\title{
A hyperspace of convex bodies arising from tensor norms
}

\author{
Luisa F. Higueras-Montaño \\ Centro de Investigación en Matemáticas (CIMAT) \\ A.P. 402 Guanajuato, Gto., México \\ fher@cimat.mx
}

\begin{abstract}
In a preceding work it is determined when a centrally symmetric convex body in $\mathbb{R}^{d}$, $d=d_{1} \cdots d_{l}$, is the closed unit ball of a reasonable crossnorm on $\mathbb{R}^{d_{1}} \otimes \cdots \otimes \mathbb{R}^{d_{l}}$. Consequently, the class of tensorial bodies is introduced, an associated tensorial BanachMazur distance is defined and the corresponding Banach-Mazur type compactum is proved to exist. In this paper, we introduce the hyperspace of these convex bodies. We called "the space of tensorial bodies". It is proved that the group of linear isomorphisms on $\mathbb{R}^{d_{1}} \otimes \cdots \otimes \mathbb{R}^{d_{l}}$ preserving decomposable vectors acts properly (in the sense of Palais) on it. A convenient compact global slice for the space is constructed. With it, topological representatives for the space of tensorial bodies and the Banach-Mazur type compactum are given. Among others, it is showed that the set of ellipsoids in the class of tensorial bodies is homeomorphic to the Euclidean space of dimension $p=\frac{d_{1}\left(d_{1}+1\right)}{2}+\cdots+\frac{d_{l}\left(d_{l}+1\right)}{2}$. We also prove that both the projective and the injective tensor products of 0 -symmetric convex bodies are continuous functions with respect to the Hausdorff distance.
\end{abstract}

Keywords: Convex body, Tensor norm, Hyperspace, Lie groups, Proper actions, Tensor product of convex sets, Linear mappings on tensor spaces, Banach-Mazur compactum.

2000 Mathematics Subject Classification: 57N20, 46M05, 52A21, 57S20, 15A69.

\section{Introduction}

Nowadays, tensor products appear as basic tools in many problems of both pure and applied nature, as can be seen in [10, 23] and the monograph [24]. In this sense, the theory of tensor products of Banach spaces, established by A. Grothendieck [17, has become in an essential tool for the study of tensor products and its applications. This can be traced by its influence to a wide range of areas, from Banach space theory [11, 12, 31] to Mathematical Analysis or Graph Theory [30] and theoretical computer science [22].

In [15], M. Fernández-Unzueta and the author give an intrinsic description of the convex bodies associated to tensor norms on finite dimensions. It means characterizing when a 0 -symmetric convex body $Q$ on $\mathbb{R}^{d}, d=d_{1} \cdots d_{l}$, is the unit ball of a reasonable crossnorm on the tensor space $\otimes_{i=1}^{l}\left(\mathbb{R}^{d_{i}},\|\cdot\|_{i}\right)$ for some norms $\|\cdot\|_{i}$ not determined a priori. See [15. Theorem 3.2]. This class of convex bodies is called tensorial bodies [15, Definition 3.3]. Among its principal properties, a Banach-Mazur type distance is introduced and its associated Banach-Mazur type compactum is exhibited [15, Theorem 3.13]. In the present 
work, we introduce the hyperspace consisting of the tensorial bodies and investigate its topological structure. The main results provide topological representatives for both the space of tensorial bodies and its associated Banach-Mazur type compactum, see Corollaries 4.9, 4.10 and 4.11.

The Banach-Mazur compactum is a central object in the study of Banach spaces [35]. It lies between two areas, namely the geometry of Banach spaces and infinite dimensional topology. Many of its metric properties, such as diameters and distances between particular points, were deeply studied in the twentieth century [16, 19, 34. In contrast, its topological structure was not determined until the beginning of this century. It was due to the works of S. Antonyan [3, 4] and S. Ageev and D. Repovš 2] that, via topological groups, the topology of the Banach-Mazur compactum was established. In this way, it is worth to notice that the results presented here extend, to the tensorial setting, many of the properties exhibited in [3] for the Banach-Mazur compactum and the hyperspace of 0-symmetric convex bodies.

Below we present the contents of the paper. We begin by stating basic results and notation from tensor norms and group actions used along the work. Then, in Section 2 , we review the main properties of the tensorial bodies [15]. A tensorial body $Q$ in $\otimes_{i=1}^{l} \mathbb{R}^{d_{i}}$ is a 0-symmetric convex body such that $Q_{1} \otimes_{\pi} \cdots \otimes_{\pi} Q_{l} \subseteq Q \subseteq Q_{1} \otimes_{\epsilon} \cdots \otimes_{\epsilon} Q_{l}$ for some 0 -symmetric convex bodies $Q_{i} \subset \mathbb{R}^{d_{i}}$. The set of tensorial bodies in $\otimes_{i=1}^{l} \mathbb{R}^{d_{i}}$ is denoted by $\mathcal{B}_{\otimes}\left(\otimes_{i=1}^{l} \mathbb{R}^{d_{i}}\right)$. Here, $\otimes_{\pi}$ and $\otimes_{\epsilon}$ are, respectively, the projective and the injective tensor product of 0-symmetric convex bodies ([7], [8, Section 4.1]).

When regarding as a hyperspace of compact convex sets in $\otimes_{i=1}^{l} \mathbb{R}^{d_{i}}$, the space of tensorial bodies $\mathcal{B}_{\otimes}\left(\otimes_{i=1}^{l} \mathbb{R}^{d_{i}}\right)$ is a topological subspace of the space of 0 -symmetric convex bodies in $\otimes_{i=1}^{l} \mathbb{R}^{d_{i}} \simeq \mathbb{R}^{d}, d=d_{1} \cdots d_{l}$. In this way, in Section 3, we prove that this space is closed and contractible, see Propositions 3.4 and 3.5. An expected and fundamental result is given in Proposition 3.3. where we show that the projective $\otimes_{\pi}$ and the injective $\otimes_{\epsilon}$ tensor products are continuous functions with respect to the Hausdorff distance.

To go further into the topological structure of the space of tensorial bodies, in Section 4. we introduce an action of a Lie group on this space. We prove that the group of linear isomorphisms on $\otimes_{i=1}^{l} \mathbb{R}^{d_{i}}$ preserving decomposable vectors $G L_{\otimes}\left(\otimes_{i=1}^{l} \mathbb{R}^{d_{i}}\right.$ ) (see Section 2 for the definition and basic proterties) acts properly (in the sense of Palais) on $\mathcal{B}_{\otimes}\left(\otimes_{i=1}^{l} \mathbb{R}^{d_{i}}\right.$ ) (Theorem 4.1). The action is given by

$$
\begin{aligned}
G L_{\otimes}\left(\otimes_{i=1}^{l} \mathbb{R}^{d_{i}}\right) \times \mathcal{B}_{\otimes}\left(\otimes_{i=1}^{l} \mathbb{R}^{d_{i}}\right) & \longrightarrow \mathcal{B}_{\otimes}\left(\otimes_{i=1}^{l} \mathbb{R}^{d_{i}}\right) \\
(T, Q) & \mapsto T Q:=\{T u: u \in Q\} .
\end{aligned}
$$

This result together with [15, Corollary 4.3] allows us to calculate the topological structure of the set of tensorial ellipsoids $\mathscr{E}_{\otimes}\left(\otimes_{i=1}^{l} \mathbb{R}^{d_{i}}\right.$ ) (i.e the ellipsoids in $\otimes_{i=1}^{l} \mathbb{R}^{d_{i}}$ that also are tensorial bodies, see Section 2.1). We show (Corollary 4.2) that:

$$
\mathscr{E}_{\otimes}\left(\otimes_{i=1}^{l} \mathbb{R}^{d_{i}}\right) \text { is homeomorphic to } \mathbb{R}^{p} \text { with } p=\frac{d_{1}\left(d_{1}+1\right)}{2}+\cdots+\frac{d_{l}\left(d_{l}+1\right)}{2} \text {. }
$$

Throughout the paper $d, d_{1}, \ldots, d_{l} \geq 2$ and $l \geq 2$ are integers. In this section, it is fundamental the subgroup $O_{\otimes}\left(\otimes_{i=1}^{l} \mathbb{R}^{d_{i}}\right):=O\left(\otimes_{i=1}^{l} \mathbb{R}^{d_{i}}\right) \cap G L_{\otimes}\left(\otimes_{i=1}^{l} \mathbb{R}^{d_{i}}\right)$ which consists of the orthogonal maps on the Hilbert tensor product $\left(\otimes_{i=1}^{l} \mathbb{R}^{d_{i}},\|\cdot\|_{H}\right)$ preserving decomposable vectors

In Section 4.1, we construct a compact $O_{\otimes}\left(\otimes_{i=1}^{l} \mathbb{R}^{d_{i}}\right)$-global slice $\mathscr{L}_{\otimes}\left(\otimes_{i=1}^{l} \mathbb{R}^{d_{i}}\right)$ for the space of tensorial bodies. To that end, we first define an equivariant retraction $l_{\otimes}: \mathcal{B}_{\otimes}\left(\otimes_{i=1}^{l} \mathbb{R}^{d_{i}}\right) \rightarrow \mathscr{E}_{\otimes}\left(\otimes_{i=1}^{l} \mathbb{R}^{d_{i}}\right)$ from the space of tensorial bodies onto the tensorial 
ellipsoids. It sends each tensorial body $Q$ in $\otimes_{i=1}^{l} \mathbb{R}^{d_{i}}$ to the Löwner ellipsoid $L \ddot{o} w\left(Q^{1} \otimes_{\pi}\right.$ $\cdots \otimes_{\pi} Q^{l}$ ) of the projective tensor product of the 0 -symmetric convex bodies $Q^{i}$ associated to $Q$ in Remark 2.3 (Proposition 4.5 ). Then, the compact $O_{\otimes}\left(\otimes_{i=1}^{l} \mathbb{R}^{d_{i}}\right.$ )-global slice of $\mathcal{B}_{\otimes}\left(\otimes_{i=1}^{l} \mathbb{R}^{d_{i}}\right)$ is the inverse image $\mathscr{L}_{\otimes}\left(\otimes_{i=1}^{l} \mathbb{R}^{d_{i}}\right)=l_{\otimes}^{-1}\left(B_{2}^{d_{1}, \ldots, d_{l}}\right)$ of the Euclidean ball $B_{2}^{d_{1}, \ldots, d_{l}}$ on $\left(\otimes_{i=1}^{l} \mathbb{R}^{d_{i}},\|\cdot\|_{H}\right)$. See Theorem 4.7 .

It is in Section 4.2 where we accomplish our principal goals: to provide topological representatives for the space of tensorial bodies (Corollaries 4.10 and 4.11) and its associated Banach-Mazur type compactum $\mathcal{B M}_{\otimes}\left(\otimes_{i=1}^{l} \mathbb{R}^{d_{i}}\right)$ (Corollary 4.9). $\mathcal{B M}_{\otimes}\left(\otimes_{i=1}^{l} \mathbb{R}^{d_{i}}\right)$ consists of the classes of tensorial bodies determined by $G L_{\otimes}\left(\otimes_{i=1}^{l} \mathbb{R}^{d_{i}}\right)$ endowed with the metric $\log \delta_{\otimes}^{B M}$ induced by the tensorial Banach-Mazur distance $\delta_{\otimes}^{B M}$, see [15, Theorem 3.13]. In Corollary 4.9, we first prove that $\mathcal{B M}_{\otimes}\left(\otimes_{i=1}^{l} \mathbb{R}^{d_{i}}\right)$ is homeomorphic to the orbit space $\mathcal{B}_{\otimes}\left(\otimes_{i=1}^{l} \mathbb{R}^{d_{i}}\right) / G L_{\otimes}\left(\otimes_{i=1}^{l} \mathbb{R}^{d_{i}}\right)$. Then, from Theorem 4.7, we obtain a homeomorphism between the compactum and the orbit space $\mathscr{L}_{\otimes}\left(\otimes_{i=1}^{l} \mathbb{R}^{d_{i}}\right) / O_{\otimes}\left(\otimes_{i=1}^{l} \mathbb{R}^{d_{i}}\right)$. We thus get the representatives:

$$
\mathcal{B} \mathcal{M}_{\otimes}\left(\otimes_{i=1}^{l} \mathbb{R}^{d_{i}}\right) \cong \mathcal{B}_{\otimes}\left(\otimes_{i=1}^{l} \mathbb{R}^{d_{i}}\right) / G L_{\otimes}\left(\otimes_{i=1}^{l} \mathbb{R}^{d_{i}}\right) \cong \mathscr{L}_{\otimes}\left(\otimes_{i=1}^{l} \mathbb{R}^{d_{i}}\right) / O_{\otimes}\left(\otimes_{i=1}^{l} \mathbb{R}^{d_{i}}\right) .
$$

This result extends to the tensorial setting Corollary 1 of [3] which exhibits new representatives for the Banach-Mazur compactum.

In Corollary 4.10, we show that the space of tensorial bodies is homeomorphic to the product $\mathscr{L}_{\otimes}\left(\otimes_{i=1}^{l} \mathbb{R}^{d_{i}}\right) \times \mathscr{E}_{\otimes}\left(\otimes_{i=1}^{l} \mathbb{R}^{d_{i}}\right)$. This, along with Corollary 4.2, allows us to prove that $\mathcal{B}_{\otimes}\left(\otimes_{i=1}^{l} \mathbb{R}^{d_{i}}\right)$ is homeomorphic to $\mathscr{L}_{\otimes}\left(\otimes_{i=1}^{l} \mathbb{R}^{d_{i}}\right) \times \mathbb{R}^{p}$ (Corollary 4.11).

We finish the paper with an appedix (Section $\mathbb{A}$ ) about the Lie group $G L_{\otimes}\left(\otimes_{i=1}^{l} \mathbb{R}^{d_{i}}\right.$ ) which may be of independent interest. We show that the subgroup $O_{\otimes}\left(\otimes_{i=1}^{l} \mathbb{R}^{d_{i}}\right)$ is a maximal compact subgroup of $G L_{\otimes}\left(\otimes_{i=1}^{l} \mathbb{R}^{d_{i}}\right.$ ) (Proposition A.2). This result, together with the so called polar decomposition theorem, allows us to determine the Lie group structure of $G L_{\otimes}\left(\otimes_{i=1}^{l} \mathbb{R}^{d_{i}}\right)$. See Proposition A.4 Corollary A.5 and Lemma A.6.

We would like to point out that this work follows the ideas presented in [3, 4], where the topological structure of the Banach-Mazur compactum is determined via the action induced by the general linear group $G L(d)$. Recent works about the topology of some hyperspaces of convex bodies are [5, 6].

\subsection{Notation}

The letters $d, d_{i}$ will denote possitive integers greater than or equal to 2 . By $\langle\cdot, \cdot\rangle,\|\cdot\|$, $B_{2}^{d}$ we denote the standard scalar product on $\mathbb{R}^{d}$ and its associated norm and Euclidean ball. As usual the group of linear isomorphisms on $\mathbb{R}^{d}$ is denoted by $G L(d)$. Every compact convex set $Q \subset \mathbb{R}^{d}$ with nonempty interior (i.e. $\operatorname{int}(Q) \neq \emptyset$ ) is called a convex body. In adition, if $Q=-Q$ then $Q$ is called a 0 -symmetric convex body. We write $\mathcal{B}(d)$ to denote the set of 0 -symmetric convex bodies in $\mathbb{R}^{d}$. For every $Q \in \mathcal{B}(d)$, its polar set, $Q^{\circ}$, is a 0 -symmetric convex body, defined as $Q^{\circ}:=\left\{y \in \mathbb{R}^{d}: \sup _{x \in Q}|\langle x, y\rangle| \leq 1\right\}$.

The Minkowski functional of a 0 -symmetric convex body $Q \subset \mathbb{R}^{d}$ is defined by $g_{Q}(x):=$ $\inf \left\{\lambda>0: \lambda^{-1} x \in Q\right\}$, for $x \in \mathbb{R}^{d}$. A well known result concerning 0 -symmetric convex bodies is the bijection between norms on $\mathbb{R}^{d}$ and 0 -symmetric convex bodies. This result, due to H. Minkowski [27], is fundamental to this work and will be used frequently without making an explicit reference. It can be stated as follows: the map $\mathcal{B}(d) \rightarrow\left\{\right.$ norms on $\left.\mathbb{R}^{d}\right\}$ that sends $Q$ to its Minkowski functional $g_{Q}(\cdot)$ is a bijection, the unit ball of $\left(\mathbb{R}^{d}, g_{Q}\right)$ is $Q$ and $g_{Q^{\circ}}(x)=\left\|\langle\cdot, x\rangle:\left(\mathbb{R}^{d}, g_{p}\right) \rightarrow \mathbb{R}\right\|$. See [32, Remark 1.7.8].

The results about convex bodies that will be used in this paper can be found in 32 . 


\subsection{Tensor norms}

We use standard notation from Banach space theory and tensor products. The symbols $M, N$ or $M_{i}$ will denote Banach spaces. The closed unit ball of $M$ will be denoted by $B_{M}$ and its dual space by $M^{*}$. We write $\mathcal{L}(M, N)$ to denote the Banach space of bounded linear operators from $M$ to $N$, with the usual operator norm.

The tensor product of $M_{i}, i=1, \ldots, l$, is denoted by $\otimes_{i=1}^{l} M_{i}$. The elements of the form $x^{1} \otimes \cdots \otimes x^{l}, x^{i} \in M_{i}$, are called decomposable vectors. For each subset $A_{i} \subseteq M_{i}$, $i=1, \ldots, l, \otimes\left(A_{1} \ldots, A_{l}\right)$ is the image of $A_{1} \times \cdots \times A_{l}$ under the canonical multilinear map $\otimes$. The decomposable vector $x_{1}^{*} \otimes \cdots \otimes x_{l}^{*}, x_{i}^{*} \in M_{i}^{*}$, determines a linear functional on $\otimes_{i=1}^{l} M_{i}$ which sends $x^{1} \otimes \cdots \otimes x^{l}$ to $x_{1}^{*}\left(x^{1}\right) \cdots x_{l}^{*}\left(x^{l}\right)$.

A norm $\alpha(\cdot)$ on the tensor product $\otimes_{i=1}^{l} M_{i}$ is a reasonable crossnorm if

1. $\alpha\left(x^{1} \otimes \cdots \otimes x^{l}\right) \leq\left\|x^{1}\right\| \cdots\left\|x^{l}\right\|$ for every $x^{i} \in M_{i}, i=1, \ldots, l$.

2. For every $x_{i}^{*} \in M_{i}^{*}$, the linear functional $x_{1}^{*} \otimes \cdots \otimes x_{l}^{*}$ is bounded and $\left\|x_{1}^{*} \otimes \cdots \otimes x_{l}^{*}\right\| \leq$ $\left\|x_{1}^{*}\right\| \cdots\left\|x_{l}^{*}\right\|$.

The biggest and the smallest reasonable crossnorms are the projective tensor norm $\pi(\cdot)$ and the injective tensor norm $\epsilon(\cdot)$ respectively. They are defined as:

$$
\begin{gathered}
\pi(u):=\inf \left\{\sum_{i=1}^{n}\left\|x_{i}^{1}\right\| \cdots\left\|x_{i}^{l}\right\|: u=\sum_{i=1}^{n} x_{i}^{1} \otimes \cdots \otimes x_{i}^{l}\right\}, \text { and } \\
\epsilon(u):=\sup \left\{\left|x_{1}^{*} \otimes \cdots \otimes x_{l}^{*}(u)\right|: x_{i}^{*} \in B_{M_{i}^{*}}, i=1, \ldots, l\right\}
\end{gathered}
$$

for $u \in \otimes_{i=1}^{l} M_{i}$. An alternative description of reasonable crossnorms, stated in terms of the norms $\pi(\cdot)$ and $\epsilon(\cdot)$, is the following: a norm $\alpha(\cdot)$ is a reasonable crossnorm if

$$
\epsilon(u) \leq \alpha(u) \leq \pi(u) \text { for every } u \in \otimes_{i=1}^{l} M_{i} .
$$

If $\alpha(\cdot)$ is a reasonable crossnorm on $\otimes_{i=1}^{l} M_{i}, \otimes_{\alpha, i=1}^{l} M_{i}$ will denote the normed space $\left(\otimes_{i=1}^{l} M_{i}, \alpha\right)$, and $M_{1} \hat{\otimes}_{\alpha} \cdots \hat{\otimes}_{\alpha} M_{l}$ its completion. For a deeper discussion about tensor norms we refer the reader to [11, 12, 17, 31].

On the tensor product of Euclidean spaces, there is natural scalar product $\langle\cdot, \cdot\rangle_{H}$. It endows $\otimes_{i=1}^{l} \mathbb{R}^{d_{i}}$ with a reasonable crossnorm $\|\cdot\|_{H} \cdot\langle\cdot, \cdot\rangle_{H}$ is defined, on decomposable vectors, as:

$$
\left\langle x^{1} \otimes \cdots \otimes x^{l}, y^{1} \otimes \cdots \otimes y^{l}\right\rangle_{H}:=\Pi_{i=1}^{l}\left\langle x^{i}, y^{i}\right\rangle
$$

and it is extended to $\otimes_{i=1}^{l} \mathbb{R}^{d_{i}}$ by multilinearity. The closed unit ball of $\otimes_{H, i=1}^{l} \mathbb{R}^{d_{i}}$ is denoted by $B_{2}^{d_{1}, \ldots, d_{l}}$. For a thorough treatment of tensor products of Euclidean spaces, we refer the reader to [20, Section 2.5].

\subsection{Group Actions}

Below we present the results from topological groups that will be used in Section 4. See [9, 28] for a deeper discussion of this topic.

All topological groups and topological spaces considered are Tychonoff. A G-space is a pair $(X, \theta)$ where $G$ is a topological group and $\theta$ is a continuous action of $G$ on $X$. If $X$ is a $G$-space and $x \in X$, then $G(x):=\{g x: g \in G\}$ denotes the orbit of $x$. By $X / G$, 
we denote the orbit space. As usual, $G_{x}:=\{g \in G: g x=x\}$ is the stabilizer of $G$ at $x$. For every subset $S \subseteq X$ and every subgroup $H \subseteq G, H(S):=\{h s: h \in H, s \in S\}$ is the $H$-saturation of $S$. If $H(S)=S, S$ is called $H$-invariant.

For any subgroup $H \subseteq G, G / H:=\{g H: g \in G\}$ is a $G$-space with the action induced by left translations.

A continuous map $f: X \rightarrow Y$ between two $G$-spaces is called equivariant or a $G$-map if $f(g x)=g f(x)$ for every $x \in X$ and $g \in G$.

A special class of actions over locally compact groups are the so called proper actions, introduced by R. Palais [29]. They enjoy many of the desirable properties of actions over compact groups, as can bee seen in [1, 29].

Let $G$ be a locally compact group and let $X$ be a Tychonoff $G$-space. The action of $G$ on $X$ is proper (in the sense of Palais) if any $x \in X$ has a small neighborhood $V$. Here, a subset $S \subseteq X$ of a $G$-space is called small if any $x \in X$ has a neighborhood $V$ such that $[S, V]:=\{g \in G: g S \cap V \neq \emptyset\}$, the transporter from $S$ to $V$, has compact closure in $G$.

Proper actions play a fundamental role for the paper. For this reason, we include some of its main properties: On every $G$-space $X$, the orbit $G(x), x \in X$, is a closed subset of $X$ and $G_{x}$ is a compact subgroup of $G$ (see [29, Proposition 1.1.4]). Moreover, there is a $G$-equivariant homeomorphism between $G / G_{x}$ and $G(x)$, see [29, Proposition 1.1.5].

Let us recall the definition of a slice:

Definition 1.1. [28, p. 305] Let $X$ be a $G$-space and $H$ a closed subgroup of $G$. An $H$-invariant subset $S \subseteq X$ is called an $H$-slice in $X$, if $G(S)$ is open in $X$ and there exists a $G$-equivariant map $f: G(S) \rightarrow G / H$ such that $S=f^{-1}(e H)$. The saturation $G(S)$ is called a tubular set. If $G(S)=X$, then $S$ is a global $H$-slice of $X$.

An important and well known result about actions of compact Lie groups $G$ establishes the existence of a $G_{x}$-slice for any $x$ in a $G$-space $X$, see [9, p. 14]. The case of non-compact Lie groups and proper actions is deeply studied in [1, 29].

\section{Tensorial Bodies}

In [15, the problem of establishing a geometric characterization of the unit balls of tensor normed spaces on finite dimensions is addressed. There, by means of the correspondence between 0 -symmetric convex bodies and norms on $\mathbb{R}^{d}, d=d_{1} \cdots d_{l}$, a characterization of the convex bodies that are closed unit balls of reasonable crossnorms on $\otimes_{i=1}^{l}\left(\mathbb{R}^{d_{i}},\|\cdot\|_{i}\right)$, for some norms (not determined a priori) on each $\mathbb{R}^{d_{i}}$ is exhibited (see [15, Theorem 3.2]). These convex bodies are the so called tensorial bodies.

Throughout the paper, $\otimes_{i=1}^{l} \mathbb{R}^{d_{i}}$ will be a Euclidean space with the scalar product $\langle\cdot, \cdot\rangle_{H}$. Given a tuple $Q_{i} \subset \mathbb{R}^{d_{i}}, i=1, \ldots, l$, of 0 -symmetric convex bodies, their projective $\otimes_{\pi}$ and injective tensor product $\otimes_{\epsilon}([7],[8$, Section 4.1]) are defined as:

$$
\begin{gathered}
Q_{1} \otimes_{\pi} \cdots \otimes_{\pi} Q_{l}:=\operatorname{conv}\left\{x^{1} \otimes \cdots \otimes x^{l} \in \otimes_{i=1}^{l} \mathbb{R}^{d_{i}}: x^{i} \in Q_{i}, i=1, \ldots, l\right\}, \text { and } \\
Q_{1} \otimes_{\epsilon} \cdots \otimes_{\epsilon} Q_{l}:=\left(Q_{1}^{\circ} \otimes_{\pi} \cdots \otimes_{\pi} Q_{l}^{\circ}\right)^{\circ} .
\end{gathered}
$$

When the normed spaces $\left(\mathbb{R}^{d_{i}}, g_{Q_{i}}\right), i=1, \ldots, l$, are considered, the projective $\otimes_{\pi}$ and the injective $\otimes_{\epsilon}$ tensor products of $Q_{1}, \ldots, Q_{l}$ are the closed unit balls associated to the projective $\pi(\cdot)$ and the injective $\epsilon(\cdot)$ tensor norms, respectively. That is,

$$
B_{\otimes_{\pi, i=1}^{l}\left(\mathbb{R}^{\left.d_{i}, g_{Q_{i}}\right)}\right.}=Q_{1} \otimes_{\pi} \cdots \otimes_{\pi} Q_{l}, \text { and }
$$




$$
B_{\otimes_{\epsilon, i=1}^{l}\left(\mathbb{R}^{\left.d_{i}, g_{Q_{i}}\right)}\right.}=Q_{1} \otimes_{\epsilon} \cdots \otimes_{\epsilon} Q_{l}
$$

See [15, pp. 5-6].

The previous relations give us the possibility to describe reasonable crossnorms in terms of convex bodies. Given a 0 -symmetric convex body $Q \subset \otimes_{i=1}^{l} \mathbb{R}^{d_{i}}, g_{Q}$ is a reasonable crossnorm on $\otimes_{i=1}^{l}\left(\mathbb{R}^{d_{i}},\|\cdot\|_{i}\right)$ if and only if

$$
Q_{1} \otimes_{\pi} \cdots \otimes_{\pi} Q_{l} \subseteq Q \subseteq Q_{1} \otimes_{\epsilon} \cdots \otimes_{\epsilon} Q_{l} .
$$

where $Q_{i}=B_{\left(\mathbb{R}^{\left.d_{i},\|\cdot\|_{i}\right)}\right.}, i=1, \ldots, l$. In this case, for every $x^{i} \in \mathbb{R}^{d_{i}}, i=1, \ldots, l$, the following hold:

$$
\begin{aligned}
g_{Q}\left(x^{1} \otimes \cdots \otimes x^{l}\right) & =g_{Q_{1}}\left(x^{1}\right) \cdots g_{Q_{l}}\left(x^{l}\right), \\
g_{Q^{\circ}}\left(x^{1} \otimes \cdots \otimes x^{l}\right) & =g_{Q_{1}^{\circ}}\left(x^{1}\right) \cdots g_{Q_{l}^{\circ}}\left(x^{l}\right),
\end{aligned}
$$

see [15, Proposition 3.1]. The inclusions in (2.1) enables the definiton of tensorial bodies:

Definition 2.1. ([15, Definition 3.3]) A 0 -symmetric convex body $Q \subset \otimes_{i=1}^{l} \mathbb{R}^{d_{i}}$ is called a tensorial body in $\otimes_{\mathbf{i}=\mathbf{1}}^{1} \mathbb{R}^{\mathbf{d}_{\mathbf{i}}}$ if there exist 0 -symmetric convex bodies $Q_{i} \subset \mathbb{R}^{d_{i}}, i=1, \ldots, l$, such that (2.1) holds.

If $Q$ satisfies (2.1), we will say that $Q$ is a tensorial body with respect to $Q_{1}, \ldots, Q_{l}$. The set of tensorial bodies in $\otimes_{i=1}^{l} \mathbb{R}^{d_{i}}$ is denoted by $\mathcal{B}_{\otimes}\left(\otimes_{i=1}^{l} \mathbb{R}^{d_{i}}\right)$. The set of tensorial bodies with respect to $Q_{1}, \ldots, Q_{l}$ is denoted by $\mathcal{B}_{Q_{1}, \ldots, Q_{l}}\left(\otimes_{i=1}^{l} \mathbb{R}^{d_{i}}\right)$.

For every non-zero decomposable vector $\mathbf{a} \in \otimes_{i=1}^{l} \mathbb{R}^{d_{i}}$ and every 0 -symmetric convex body $Q \subset \otimes_{i=1}^{l} \mathbb{R}^{d_{i}}$, if $\mathbf{a}=a^{1} \otimes \cdots \otimes a^{l}$ then $Q_{i}^{a^{1}, \ldots, a^{l}}, i=1, \ldots, l$, defined as

$$
Q_{i}^{a^{1}, \ldots, a^{l}}:=\left\{x^{i} \in \mathbb{R}^{d_{i}}: a^{1} \otimes \cdots \otimes a^{i-1} \otimes x^{i} \otimes a^{i+1} \otimes \cdots \otimes a^{l} \in Q\right\},
$$

is a 0 -symmetric convex body. The next theorem establishes when a 0 -symmetric convex body is the closed unit ball of a reasonable crossnorm for some norms, not determined a priori, on each $\mathbb{R}^{d_{i}}$.

Theorem 2.2. ([15, Corollary 3.4]) Let $Q \subset \otimes_{i=1}^{l} \mathbb{R}^{d_{i}}$ be a 0 -symmetric convex body. The following are equivalent:

1. $Q$ is a tensorial body in $\otimes_{i=1}^{l} \mathbb{R}^{d_{i}}$.

2. There exist norms $\|\cdot\|_{i}$ on $\mathbb{R}^{d_{i}}, i=1, \ldots, l$, such that $g_{Q}$ is a reasonable crossnorm on $\otimes_{i=1}^{l}\left(\mathbb{R}^{d_{i}},\|\cdot\|_{i}\right)$.

3. For any $a^{1} \otimes \cdots \otimes a^{l} \in \partial Q$,

$$
Q_{1}^{a^{1}, \ldots, a^{l}} \otimes_{\pi} \cdots \otimes_{\pi} Q_{l}^{a^{1}, \ldots, a^{l}} \subseteq Q \subseteq Q_{1}^{a^{1}, \ldots, a^{l}} \otimes_{\epsilon} \cdots \otimes_{\epsilon} Q_{l}^{a^{1}, \ldots, a^{l}} .
$$

In this case, $g_{Q_{i}^{a^{1}, \ldots, a^{l}}}(\cdot)=\frac{1}{\left\|a^{i}\right\|_{i}}\|\cdot\|_{i}$ for $i=1, \ldots, l$.

Remark 2.3. To simplify many arguments in the forthcoming proofs, it is convenient to choose the convex bodies of (2.4) in a specific way: for every 0 -symmetric convex body $Q \subset$ $\otimes_{i=1}^{l} \mathbb{R}^{d_{i}}, Q^{i}$ denote the convex bodies generated by $e_{1}^{d_{1}} \otimes \cdots \otimes\left(\lambda e_{1}^{d_{l}}\right), \lambda=\frac{1}{g_{Q}\left(e_{1}^{d_{1}} \otimes \cdots \otimes e_{1}^{d_{l}}\right)}$. That is, $Q^{i}:=Q_{i}^{e_{1}^{d_{1}}, \ldots, \lambda e_{l}^{d_{l}}}$ for $i=1, \ldots, l$. 
In [15. Section 3.2], it is proved that there exists a Banach-Mazur type distance on the set of tensorial bodies. It is called the tensorial Banach-Mazur distance. Its existence follows from the relation between tensorial bodies and linear mappings preserving decomposable vectors that we describe below.

A linear map $T: \otimes_{i=1}^{l} \mathbb{R}^{d_{i}} \rightarrow \otimes_{i=1}^{l} \mathbb{R}^{d_{i}}$ preserves decomposable vectors if $T\left(x^{1} \otimes \cdots \otimes x^{l}\right)$ is a decomposable vector for any $x^{i} \in \mathbb{R}^{d_{i}}, i=1, \ldots, l$. The set of linear isomorphisms preserving decomposabe vectors is denoted by $G L_{\otimes}\left(\otimes_{i=1}^{l} \mathbb{R}^{d_{i}}\right)$.

In [25, Corollary 2.14], it is proved that for every $T \in G L_{\otimes}\left(\otimes_{i=1}^{l} \mathbb{R}^{d_{i}}\right)$ and every $x^{i} \in \mathbb{R}^{d_{i}}$, we have:

$$
T\left(x^{1} \otimes \cdots \otimes x^{l}\right)=T_{1}\left(x^{\sigma(1)}\right) \otimes \cdots \otimes T_{l}\left(x^{\sigma(l)}\right)
$$

where $\sigma$ is a permutation on $\{1, \ldots, l\}$ and $T_{i} \in G L\left(d_{i}\right)$ for $i=1, \ldots, l$. The latter along with [15, Theorem 3.12] allows to prove that for every $T \in G L_{\otimes}\left(\otimes_{i=1}^{l} \mathbb{R}^{d_{i}}\right)$ :

$$
\text { If } Q \in \mathcal{B}_{Q_{1}, \ldots, Q_{l}}\left(\otimes_{i=1}^{l} \mathbb{R}^{d_{i}}\right) \text {, then } T Q \in \mathcal{B}_{T_{1} Q_{\sigma(1)}, \ldots, T_{l} Q_{\sigma(l)}}\left(\otimes_{i=1}^{l} \mathbb{R}^{d_{i}}\right) \text {. }
$$

In this case, for every tuple of 0 -symmetric convex bodies $Q_{i} \subset \mathbb{R}^{d_{i}}, i=1, \ldots, l$, and $\alpha=\pi, \epsilon$ it holds

$$
T\left(Q_{1} \otimes_{\alpha} \cdots \otimes_{\alpha} Q_{l}\right)=T_{1}\left(Q_{\sigma(1)}\right) \otimes_{\alpha} \cdots \otimes_{\alpha} T_{l}\left(Q_{\sigma(l)}\right) .
$$

The tensorial Banach-Mazur distance $\delta_{\otimes}^{B M}(P, Q)$, between tensorial bodies $P, Q \subset$ $\otimes_{i=1}^{l} \mathbb{R}^{d_{i}}$, is defined as:

$$
\delta_{\otimes}^{B M}(P, Q):=\inf \left\{\lambda \geq 1: Q \subseteq T P \subseteq \lambda Q, \text { for } T \in G L_{\otimes}\left(\otimes_{i=1}^{l} \mathbb{R}^{d_{i}}\right)\right\} .
$$

In [15, Section 3.2], it is proved that for each pair $P, Q \in \mathcal{B}_{\otimes}\left(\otimes_{i=1}^{l} \mathbb{R}^{d_{i}}\right)$, the infimum in (2.8) attains its value at some $\lambda \geq 1$ and $T \in G L_{\otimes}\left(\otimes_{i=1}^{l} \mathbb{R}^{d_{i}}\right)$. This naturally leads to the following equivalence relation: For every pair of tensorial bodies $P, Q \subset \otimes_{i=1}^{l} \mathbb{R}^{d_{i}}, P \sim Q$ if and only if $\delta_{\otimes}^{B M}(P, Q)=1$ or, equivalently, there exists $T \in G L_{\otimes}\left(\otimes_{i=1}^{l} \mathbb{R}^{d_{i}}\right)$ such that $T(P)=Q$.

The set of equivalence classes determined by this relation is denoted by $\mathcal{B} \mathcal{M}_{\otimes}\left(\otimes_{i=1}^{l} \mathbb{R}^{d_{i}}\right)$. In [15, Theorem 3.13], it is showed that $\left(\mathcal{B M}_{\otimes}\left(\otimes_{i=1}^{l} \mathbb{R}^{d_{i}}\right), \log \delta_{\otimes}^{B M}\right)$ is a compact metric space. It is called the compactum of tensorial bodies.

\subsection{Tensorial ellipsoids}

An ellipsoid $\mathcal{E} \subset V$ in a $d$-dimensional vector space is defined as the image of the Euclidean ball $B_{2}^{d}$ by a linear isomorphism $T: \mathbb{R}^{d} \rightarrow V$. In the case of ellipsoids in $\otimes_{i=1}^{l} \mathbb{R}^{d_{i}}$, since we have fixed the scalar product $\langle\cdot, \cdot\rangle_{H}$, we say that $\mathcal{E} \subset \otimes_{i=1}^{l} \mathbb{R}^{d_{i}}$ is an ellipsoid if $\mathcal{E}=$ $T\left(B_{2}^{d_{1}, \ldots, d_{l}}\right)$ for some linear isomorphism $T: \otimes_{i=1}^{l} \mathbb{R}^{d_{i}} \rightarrow \otimes_{i=1}^{l} \mathbb{R}^{d_{i}}$.

An ellipsoid $\mathcal{E} \subset \otimes_{i=1}^{l} \mathbb{R}^{d_{i}}$ is a tensorial ellipsoid if $\mathcal{E}$ is also a tensorial body in $\otimes_{i=1}^{l} \mathbb{R}^{d_{i}}$. The set of tensorial ellipsoids in $\otimes_{i=1}^{l} \mathbb{R}^{d_{i}}$ is denoted by $\mathscr{E}_{\otimes}\left(\otimes_{i=1}^{l} \mathbb{R}^{d_{i}}\right)$. The Hilbertian tensor product of ellipsoids $\mathcal{E}_{i}, i=1, \ldots, l$, introduced in [7], is defined as:

$$
\mathcal{E}_{1} \otimes_{2} \cdots \otimes_{2} \mathcal{E}_{l}:=T_{1} \otimes \cdots \otimes T_{l}\left(B_{2}^{d_{1}, \ldots, d_{l}}\right),
$$

for $\mathcal{E}_{i}=T_{i}\left(B_{2}^{d_{i}}\right)$. It does not depend on the election of the maps $T_{i}$. The product $\otimes_{2}$ gives examples of tensorial ellipsoids. In this case, $\mathcal{E}_{1} \otimes_{2} \cdots \otimes_{2} \mathcal{E}_{l}$ is the closed unit ball of the 
Hilbert tensor product $\otimes_{H, i=1}^{l}\left(\mathbb{R}^{d_{i}}, g_{\mathcal{E}_{i}}\right)$. In particular, the Euclidean ball $B_{2}^{d_{1}, \ldots, d_{l}}$ is such that

$$
B_{2}^{d_{1}, \ldots, d_{l}}=B_{2}^{d_{1}} \otimes_{2} \cdots \otimes_{2} B_{2}^{d_{l}} .
$$

In [15, Section 4], the ellipsoids in the class of tensorial bodies are completely described. It is proved that if $\mathcal{E}$ is a tensorial ellipsoid in $\otimes_{i=1}^{l} \mathbb{R}^{d_{i}}$, then there exist $T_{i} \in G L\left(d_{i}\right)$, $i=1, \ldots, l$, such that

$$
\mathcal{E}=T_{1} \otimes \cdots \otimes T_{l}\left(B_{2}^{d_{1}, \ldots, d_{l}}\right)=T_{1}\left(B_{2}^{d_{1}}\right) \otimes_{2} \cdots \otimes_{2} T_{l}\left(B_{2}^{d_{l}}\right) .
$$

This shows that tensorial ellipsoids are the image of $B_{2}^{d_{1}, \ldots, d_{l}}$ by elements of $G L_{\otimes}\left(\otimes_{i=1}^{l} \mathbb{R}^{d_{i}}\right)$. See [15, Corollary 4.3].

\section{$3 \quad$ The space of tensorial bodies $\mathcal{B}_{\otimes}\left(\otimes_{i=1}^{l} \mathbb{R}^{d_{i}}\right)$}

For every pair $P, Q$ of non-empty compact sets contained in a Euclidean space $\mathbb{E}$, the Hausdorff distance $\delta^{H}(P, Q)$ is defined as:

$$
\delta^{H}(P, Q):=\max \left\{\operatorname{supinf}_{x \in P}\|x-y\|_{\mathbb{E}}, \sup _{y \in Q} \inf _{x \in P}\|y-x\|_{\mathbb{E}}\right\}
$$

or, equivalently, by $\delta^{H}(P, Q)=\min \left\{\lambda \geq 0: P \subseteq Q+\lambda B_{\mathbb{E}}, Q \subseteq P+\lambda B_{\mathbb{E}}\right\}$. If $P, Q$ are, in addition, 0-symmetric convex bodies, then we have the following well known characterization of $\delta^{H}$, see [32, Theorem 1.8.11]:

$$
\delta^{H}(P, Q)=\sup _{x \in \partial B_{\mathbb{E}}}\left|g_{P^{\circ}}(x)-g_{Q^{\circ}}(x)\right| .
$$

Below, we prove that both the projective $\otimes_{\pi}$ and the injective $\otimes_{\epsilon}$ tensor products of 0 -symmetric convex bodies are continuous functions with respect to $\delta^{H}$ (Proposition 3.3).

Lemma 3.1. For every sequence $\left\{C_{n}\right\}_{n \in \mathbb{N}} \subset \mathcal{B}(d)$ and $C \in \mathcal{B}(d)$, we have:

1. If $g_{C_{n}}(\cdot)$ converges uniformly on $\partial B_{2}^{d}$ to $g_{C}(\cdot)$, then the same holds for $g_{C_{n}^{\circ}}(\cdot)$ and $g_{C^{\circ}}(\cdot)$.

2. If $C_{n}$ converges to $C$, in the Hausdorff distance, then $g_{C_{n}}(\cdot)$ converges uniformly on $\partial B_{2}^{d}$ to $g_{C}(\cdot)$. In particular, $C_{n}^{\circ}$ goes to $C^{\circ}$ in the Hausdorff distance.

We do not include the proof of Lemma 3.1, because it can be directly proved by using (3.1). For each $d \in \mathbb{N}$, the function

$$
\begin{aligned}
\nu:\left(\mathcal{B}(d), \delta^{H}\right) & \rightarrow \mathbb{R} \\
Q & \mapsto \nu(Q)=\sup _{x \in Q}\|x\|
\end{aligned}
$$

is uniformly continuous, see [5, Lemma 4.2].

Lemma 3.2. Let $P_{i}, Q_{i} \subset \mathbb{R}^{d_{i}}, i=1, \ldots, l$, be 0 -symmetric convex bodies. Then, for each $i$, the following holds:

$$
\delta^{H}\left(Q_{1} \otimes_{\pi} \cdots \otimes_{\pi} Q_{i} \otimes_{\pi} \cdots \otimes_{\pi} Q_{l}, Q_{1} \otimes_{\pi} \cdots \otimes_{\pi} P_{i} \otimes_{\pi} \cdots \otimes_{\pi} Q_{l}\right) \leq \delta^{H}\left(Q_{i}, P_{i}\right) \prod_{j \neq i} \nu_{j}\left(Q_{j}\right) .
$$

Here, $\nu_{j}: \mathcal{B}\left(d_{j}\right) \rightarrow \mathbb{R}$ is the map of (3.2). 
Proof. Let us fix $i \in\{1, \ldots, l\}$. Observe that from the definition of $\otimes_{\pi}$ and the properties of the Hausdorff distance [32, p. 51], it follows that

$$
\begin{gathered}
\delta^{H}\left(Q_{1} \otimes_{\pi} \cdots \otimes_{\pi} Q_{i} \otimes_{\pi} \cdots \otimes_{\pi} Q_{l}, Q_{1} \otimes_{\pi} \cdots \otimes_{\pi} P_{i} \otimes_{\pi} \cdots \otimes_{\pi} Q_{l}\right) \leq \\
\delta^{H}\left(\otimes\left(Q_{1}, \ldots, Q_{i}, \ldots, Q_{l}\right), \otimes\left(Q_{1}, \ldots, P_{i}, \ldots, Q_{l}\right)\right) .
\end{gathered}
$$

On the other hand, if we take $\lambda \geq \delta^{H}\left(P_{i}, Q_{i}\right)$ then $P_{i} \subseteq Q_{i}+\lambda B_{2}^{d_{i}}$ and $Q_{i} \subseteq P_{i}+\lambda B_{2}^{d_{i}}$. Thus, for every $x^{i} \in Q_{i}$, there exists $y^{i} \in P_{i}$ and $u_{i} \in B_{2}^{d_{i}}$ such that $x^{i}=y^{i}+\lambda u_{i}$. Hence, for every $x^{j} \in Q_{j}, j=1, \ldots, i, \ldots, l$, one has

$$
\begin{aligned}
x^{1} \otimes \cdots \otimes x^{i} \otimes \cdots \otimes x^{l} & =x^{1} \otimes \cdots \otimes\left(y^{i}+\lambda u_{i}\right) \otimes \cdots \otimes x^{l} \\
& =x^{1} \otimes \cdots \otimes y^{i} \otimes \cdots \otimes x^{l}+x^{1} \otimes \cdots \otimes \lambda u_{i} \otimes \cdots \otimes x^{l} .
\end{aligned}
$$

Since $x^{1} \otimes \cdots \otimes y^{i} \otimes \cdots \otimes x^{l} \in \otimes\left(Q_{1}, \ldots, P_{i}, \ldots, Q_{l}\right), x^{j} \in \nu_{j}\left(Q_{j}\right) B_{2}^{d_{j}}$ for $j \neq i$, then by (2.9), we have

$$
\otimes\left(Q_{1}, \ldots, Q_{i}, \ldots, Q_{l}\right) \subseteq \otimes\left(Q_{1}, \ldots, P_{i}, \ldots, Q_{l}\right)+\lambda \prod_{j \neq i} \nu_{j}\left(Q_{j}\right) B_{2}^{d_{1}, \ldots, d_{l}} .
$$

In a similar way, the above inclusion also holds if we exchange $\otimes\left(Q_{1}, \ldots, Q_{i}, \ldots, Q_{l}\right)$ and $\otimes\left(Q_{1}, \ldots, P_{i}, \ldots, Q_{l}\right)$. Therefore,

$$
\delta^{H}\left(\otimes\left(Q_{1}, \ldots, Q_{i}, \ldots, Q_{l}\right), \otimes\left(Q_{1}, \ldots, P_{i}, \ldots, Q_{l}\right)\right) \leq \lambda \prod_{j \neq i} \nu_{j}\left(Q_{j}\right)
$$

and the result then follows from (3.3).

Proposition 3.3. The projective $\otimes_{\pi}$ and the injective $\otimes_{\epsilon}$ tensor products are continuous functions. That is, for $\alpha=\pi, \epsilon$,

$$
\begin{aligned}
\otimes_{\alpha}:\left(\mathcal{B}\left(d_{1}\right), \delta^{H}\right) \times \cdots \times\left(\mathcal{B}\left(d_{l}\right), \delta^{H}\right) & \rightarrow\left(\mathcal{B}\left(\otimes_{i=1}^{l} \mathbb{R}^{d_{i}}\right), \delta^{H}\right) \\
\left(Q_{1}, \ldots, Q_{l}\right) & \mapsto Q_{1} \otimes_{\alpha} \cdots \otimes_{\alpha} Q_{l}
\end{aligned}
$$

is continuous.

Proof. First we prove that $\otimes_{\pi}$ is continuous. To that end for each $i=1, \ldots, l$, let $\left\{Q_{i}^{n}\right\}_{n \in \mathbb{N}}$ be a sequence in $\mathcal{B}\left(d_{i}\right)$ converging to $Q_{i} \in \mathcal{B}\left(d_{i}\right)$. From the triangle inequality and Lemma 3.2. we have

$$
\begin{gathered}
\delta^{H}\left(Q_{1} \otimes_{\pi} \cdots \otimes_{\pi} Q_{l}, Q_{1}^{n} \otimes_{\pi} \cdots \otimes_{\pi} Q_{l}^{n}\right) \leq \\
\sum_{i=1}^{l} \delta^{H}\left(Q_{1}^{n} \otimes_{\pi} \cdots \otimes_{\pi} Q_{i-1}^{n} \otimes_{\pi} Q_{i} \otimes_{\pi} Q_{i+1} \otimes_{\pi} \cdots \otimes_{\pi} Q_{l},\right. \\
\left.Q_{1}^{n} \otimes_{\pi} \cdots \otimes_{\pi} Q_{i-1}^{n} \otimes_{\pi} Q_{i}^{n} \otimes_{\pi} Q_{i+1} \otimes_{\pi} \cdots \otimes_{\pi} Q_{l}\right) \leq \\
\sum_{i=1}^{l} \delta^{H}\left(Q_{i}, Q_{i}^{n}\right) \prod_{j<i} \nu_{j}\left(Q_{j}^{n}\right) \prod_{j>i} \nu_{j}\left(Q_{j}\right) .
\end{gathered}
$$

Thus, by the continuity of $\nu_{i}$ ([5, Lemma 4.2]) and the fact that $Q_{i}^{n}$ converges to $Q_{i}$, it follows that $\delta^{H}\left(Q_{1} \otimes_{\pi} \cdots \otimes_{\pi} Q_{l}, Q_{1}^{n} \otimes_{\pi} \cdots \otimes_{\pi} Q_{l}^{n}\right)$ goes to 0 . This proves the continuity of $\otimes_{\pi}$.

To prove that $\otimes_{\epsilon}$ is continuous, observe that if $Q_{i}^{n}$ and $Q_{i}$ are as above, then, by Lemma 3.1. $\left(Q_{i}^{n}\right)^{\circ}$ converges to $Q_{i}^{\circ}$. Thus, by the continuity of $\otimes_{\pi},\left(Q_{1}^{n}\right)^{\circ} \otimes_{\pi} \cdots \otimes_{\pi}\left(Q_{l}^{n}\right)^{\circ}$ converges to $Q_{1}^{\circ} \otimes_{\pi} \cdots \otimes_{\pi} Q_{l}^{\circ}$. Hence, from Lemma 3.1. $\left(\left(Q_{1}^{n}\right)^{\circ} \otimes_{\pi} \cdots \otimes_{\pi}\left(Q_{l}^{n}\right)^{\circ}\right)^{\circ}$ converges to $\left(Q_{1}^{\circ} \otimes_{\pi} \cdots \otimes_{\pi} Q_{l}^{\circ}\right)^{\circ}$. This shows that $\delta^{H}\left(Q_{1}^{n} \otimes_{\epsilon} \cdots \otimes_{\epsilon} Q_{l}^{n}, Q_{1} \otimes_{\epsilon} \cdots \otimes_{\epsilon} Q_{l}\right)$ goes to 0 , and so the continuity of $\otimes_{\epsilon}$ is proved. 


\subsection{Basic Properties}

From now on, we will refer to $\mathcal{B}_{\otimes}\left(\otimes_{i=1}^{l} \mathbb{R}^{d_{i}}\right)$ as the space of tensorial bodies. Unless otherwise state the topology on it will be the one determined by the Hausdorff distance. In this way, $\mathcal{B}_{\otimes}\left(\otimes_{i=1}^{l} \mathbb{R}^{d_{i}}\right)$ is a topological subspace of the space of 0 -symmetric convex bodies in $\otimes_{i=1}^{l} \mathbb{R}^{d_{i}}$. Here we will prove that it is a closed and contractible (topological) subspace. See Propositions 3.4 and 3.5

To shorten notation, when no confusion can arise, we write simply $\mathcal{B}_{\otimes}$ or $\mathcal{B}_{Q_{1}, \ldots, Q_{l}}$ instead of $\mathcal{B}_{\otimes}\left(\otimes_{i=1}^{l} \mathbb{R}^{d_{i}}\right)$ and $\mathcal{B}_{Q_{1}, \ldots, Q_{l}}\left(\otimes_{i=1}^{l} \mathbb{R}^{d_{i}}\right)$. Recall that for every 0 -symmetric convex body $Q, g_{Q}$ denotes its Minkowski functional. Also, if $Q \subset \otimes_{i=1}^{l} \mathbb{R}^{d_{i}}$ then $Q^{i}, i=1, \ldots, l$, are the 0 -symmetric convex bodies of Remark 2.3 .

Proposition 3.4. The following statements hold:

1. Let $Q_{i} \subset \mathbb{R}^{d_{i}}, i=1, \ldots, l$, be 0 -symmetric convex bodies. Then $\mathcal{B}_{Q_{1}, \ldots, Q_{l}}\left(\otimes_{i=1}^{l} \mathbb{R}^{d_{i}}\right)$ is a compact convex subset of the space of 0 -symmetric convex bodies in $\otimes_{i=1}^{l} \mathbb{R}^{d_{i}}$.

2. $\mathcal{B}_{\otimes}\left(\otimes_{i=1}^{l} \mathbb{R}^{d_{i}}\right)$ is closed in the space of 0 -symmetric convex bodies in $\otimes_{i=1}^{l} \mathbb{R}^{d_{i}}$.

Proof. (1) The convexity of $\mathcal{B}_{Q_{1}, \ldots, Q_{l}}$ follows directly from the properties of the Minkowski sum. In this sense, for any convex body and any $0 \leq t \leq 1$. If $\alpha=\pi, \epsilon$ then $t Q_{1} \otimes_{\alpha} \cdots \otimes_{\alpha}$ $Q_{l}+(1-t) Q_{1} \otimes_{\alpha} \cdots \otimes_{\alpha} Q_{l}=Q_{1} \otimes_{\alpha} \cdots \otimes_{\alpha} Q_{l}$. Hence, by (2.1), if $P, Q \in \mathcal{B}_{Q_{1}, \ldots, Q_{l}}$, then $t Q+(1-t) P \in \mathcal{B}_{Q_{1}, \ldots, Q_{l}}$.

To prove that $\mathcal{B}_{Q_{1}, \ldots, Q_{l}}$ is compact, it is enough to show that it is closed. The result then follows by the Blaschke selection theorem [32, Theorem 1.8.6] and the fact that $\mathcal{B}_{Q_{1}, \ldots, Q_{l}}$ is bounded (every element is contained in $Q_{1} \otimes_{\epsilon} \cdots \otimes_{\epsilon} Q_{l}$ ).

Let $P_{n} \in \mathcal{B}_{Q_{1}, \ldots, Q_{l}}$ be a sequence converging to a 0 -symmetric convex body $P$. We will see that $P$ is a tensorial body in $\mathcal{B}_{Q_{1}, \ldots, Q_{l}}$. By (2) of Lemma 3.1 we have:

$$
\begin{aligned}
g_{P}\left(x^{1} \otimes \cdots \otimes x^{l}\right)=\lim _{n \rightarrow \infty} g_{P_{n}}\left(x^{1} \otimes \cdots \otimes x^{l}\right) & \stackrel{*}{=} \lim _{n \rightarrow \infty} g_{Q_{1}}\left(x^{1}\right) \cdots g_{Q_{l}}\left(x^{l}\right) \\
& =g_{Q_{1}}\left(x^{1}\right) \cdots g_{Q_{l}}\left(x^{l}\right) .
\end{aligned}
$$

(*) follows from the fact that each $P_{n} \in \mathcal{B}_{Q_{1}, \ldots, Q_{l}}$, see (2.2). Similar arguments show that $g_{P^{\circ}}\left(x^{1} \otimes \cdots \otimes x^{l}\right)=g_{\left(Q_{1}\right)^{\circ}}\left(x^{1}\right) \cdots g_{\left(Q_{l}\right)^{\circ}}\left(x^{l}\right)$. Therefore, by [15, Proposition 3.1], $P \in \mathcal{B}_{Q_{1}, \ldots, Q_{l}}$.

(2) The closedness of $\mathcal{B}_{\otimes}$ follows directly from [15, Proposition 3.7]. To see this, take a sequence $Q_{n} \in \mathcal{B}_{\otimes}$ converging to a 0 -symmetric convex body $Q$. By Lemma 3.1, $g_{Q_{n}}$ converges uniformly on compact sets to $g_{Q}$. Thus, from [15, Proposition 3.7], $Q$ must be a tensorial body.

Proposition 3.5. The space of tensorial bodies $\mathcal{B}_{\otimes}\left(\otimes_{i=1}^{l} \mathbb{R}^{d_{i}}\right)$ is contractible.

Proof. We will define a contracting homotopy from $\mathcal{B}_{\otimes}$ to $B_{2}^{d_{1}} \otimes_{\pi} \cdots \otimes_{\pi} B_{2}^{d_{l}}$. For every tensorial body $Q \subset \otimes_{i=1}^{l} \mathbb{R}^{d_{i}}$ and every real number $t \in[0,1]$, let $H$ be defined as:

$$
\begin{gathered}
H(Q, t)=(1-2 t) Q+2 t\left(Q^{1} \otimes_{\pi} \cdots \otimes_{\pi} Q^{l}\right) \text { for } 0 \leq t \leq \frac{1}{2}, \text { and } \\
H(Q, t)=\left((2-2 t) Q^{1}+(2 t-1) B_{2}^{d_{1}}\right) \otimes_{\pi} \cdots \otimes_{\pi}\left((2-2 t) Q^{l}+(2 t-1) B_{2}^{d_{l}}\right),
\end{gathered}
$$


for $\frac{1}{2} \leq t \leq 1$. Below, we show that $H: \mathcal{B}_{\otimes} \times[0,1] \rightarrow \mathcal{B}_{\otimes}$ is the desired homotopy.

To prove that it is well defined, observe that since every tensorial body $Q$ belongs to its corresponding $\mathcal{B}_{Q^{1}, \ldots, Q^{l}}$, then, by the convexity of $\mathcal{B}_{Q^{1}, \ldots, Q^{l}}$ (Proposition 3.4), it holds that $(1-2 t) Q+2 t\left(Q^{1} \otimes_{\pi} \cdots \otimes_{\pi} Q^{l}\right)$ is a tensorial body for all $t \in\left[0, \frac{1}{2}\right]$. Also, by definition of $H, H(Q, t)$ is a tensorial body for $\frac{1}{2} \leq t \leq 1$. Clearly $H(Q, 0)$ is the identity map on $\mathcal{B}_{\otimes}$ and $H(Q, 1)=B_{2}^{d_{1}} \otimes_{\pi} \cdots \otimes_{\pi} B_{2}^{d_{l}}$.

It remains to prove the continuity of $H$. To this end, let $t_{n}$ and $Q_{n}$ be sequences of both real numbers in $\left[0, \frac{1}{2}\right]$ and of tensorial bodies converging to $t$ and $Q$ respectively. From Lemma 3.1 and [15, Proposition 3.7], $g_{Q_{n}^{i}}$ converges uniformly (on compact sets) to $g_{Q^{i}}$, for each $i=1, \ldots, l$. This, along with (3.1) and (1) in Lemma 3.1 proves that $Q_{n}^{i}$ goes to $Q^{i}$, for each $i$. Hence, by the continuity of $\otimes_{\pi}, Q_{n}^{1} \otimes_{\pi} \cdots \otimes_{\pi} Q_{n}^{l}$ converges to $Q^{1} \otimes_{\pi} \cdots \otimes_{\pi} Q^{l}$ in the Hausdorff distance. Now, by the triangle inequality, we have:

$$
\begin{gathered}
\delta^{H}\left(H\left(Q_{n}, t_{n}\right), H(Q, t)\right) \leq \delta^{H}\left(H\left(Q_{n}, t_{n}\right), H\left(Q, t_{n}\right)\right)+\delta^{H}\left(H\left(Q, t_{n}\right), H(Q, t)\right) \\
\leq\left(1-2 t_{n}\right) \delta^{H}\left(Q_{n}, Q\right)+2 t_{n} \delta^{H}\left(Q_{n}^{1} \otimes_{\pi} \cdots \otimes_{\pi} Q_{n}^{l}, Q^{1} \otimes_{\pi} \cdots \otimes_{\pi} Q^{l}\right) \\
+2\left|t_{n}-t\right|\left(\nu(Q)+\nu\left(Q^{1} \otimes_{\pi} \cdots \otimes_{\pi} Q^{l}\right)\right),
\end{gathered}
$$

where $\nu$ is the map defined in (3.2). The convergence of $H\left(Q_{n}, t_{n}\right)$ to $H(Q, t)$ then follows from the last inequality and the previous discussion. In the same manner, the continuity on $\left[\frac{1}{2}, 1\right]$ follows from the continuity of both the projective tensor product $\otimes_{\pi}$ and the map $t \mapsto(2-2 t) Q^{i}+(2 t-1) B_{2}^{d_{i}}, i=1, \ldots, l$.

We finish this section with two results that will be used in the proof of Theorem 4.1 .

Lemma 3.6. Let $\varepsilon>0$ and let $P \subset \otimes_{i=1}^{l} \mathbb{R}^{d_{i}}$ be a tensorial body such that $2 \varepsilon B_{2}^{d_{1}, \ldots, d_{l}} \subseteq P$. If $\delta^{H}(P, Q)<\varepsilon$, for some tensorial body $Q$, then $\varepsilon B_{2}^{d_{1}, \ldots, d_{l}} \subseteq Q$.

We do not include the proof of Lemma 3.6 because it follows from [5, Lemma 3.1] by making $x_{0}$ equals to zero.

Lemma 3.7. Let $\varepsilon>0$ and let $P \subset \otimes_{i=1}^{l} \mathbb{R}^{d_{i}}$ be a tensorial body such that $2 \varepsilon B_{2}^{d_{1}, \ldots, d_{l}} \subseteq P$. Then the set, $V_{P}(\varepsilon):=\left\{Q \in \mathcal{B}_{\otimes}\left(\otimes_{i=1}^{l} \mathbb{R}^{d_{i}}\right): \delta^{H}(P, Q)<\varepsilon\right\}$ is a relatively compact set in the space of tensorial bodies.

Proof. We will prove that each sequence in $V_{P}(\varepsilon)$ has a convergent subsequence in $\mathcal{B}_{\otimes}$. Suppose that $2 \varepsilon B_{2}^{d_{1}, \ldots, d_{l}} \subseteq P$ and let $Q_{k}, k \in \mathbb{N}$, be a sequence contained in $V_{P}(\varepsilon)$. Clearly $Q_{k}, k \in \mathbb{N}$, is a bounded sequence of non-empty compact convex sets. Thus, from the Blaschke selection theorem ([32, Theorem 1.8.6]) there exists a subsequence $Q_{k_{i}}$ such that $Q_{k_{i}}$ converges to a non-empty compact convex set $Q \subset \otimes_{i=1}^{l} \mathbb{R}^{d_{i}}$. By Lemma 3.6, $\varepsilon B_{2}^{d_{1}, \ldots, d_{l}} \subseteq Q_{k_{i}}$ for all $i \in \mathbb{N}$, therefore $\varepsilon B_{2}^{d_{1}, \ldots, d_{l}} \subseteq Q$ and $Q$ is a 0 -symmetric convex body. Hence, from Proposition 3.4 it follows that $Q \in \mathcal{B}_{\otimes}$. This completes the proof.

\section{A natural action on the space of tensorial bodies $\mathcal{B}_{\otimes}\left(\otimes_{i=1}^{l} \mathbb{R}^{d_{i}}\right)$}

Recall that $G L_{\otimes}\left(\otimes_{i=1}^{l} \mathbb{R}^{d_{i}}\right)$ is the set of linear isomorphisms on $\otimes_{i=1}^{l} \mathbb{R}_{i}^{d}$ that preseve decomposable vectors (see Section 2). In a similar way, $O_{\otimes}\left(\otimes_{i=1}^{l} \mathbb{R}^{d_{i}}\right)$ consists of the orthogonal maps on $\otimes_{i=1}^{l} \mathbb{R}^{d_{i}}$ which enjoy of the same property. That is,

$$
O_{\otimes}\left(\otimes_{i=1}^{l} \mathbb{R}^{d_{i}}\right):=G L_{\otimes}\left(\otimes_{i=1}^{l} \mathbb{R}^{d_{i}}\right) \cap O\left(\otimes_{i=1}^{l} \mathbb{R}^{d_{i}}\right) .
$$


In [15, Proposition 3.11 and Theorem 3.12], it is proved that $G L_{\otimes}\left(\otimes_{i=1}^{l} \mathbb{R}^{d_{i}}\right)$ is a closed subgroup of $G L\left(\otimes_{i=1}^{l} \mathbb{R}^{d_{i}}\right)$ which preserves tensorial bodies, see also (2.6). This allows us to define a natural action of $G L_{\otimes}\left(\otimes_{i=1}^{l} \mathbb{R}^{d_{i}}\right)$ on the space $\mathcal{B}_{\otimes}\left(\otimes_{i=1}^{l} \mathbb{R}^{d_{i}}\right)$. It is given by:

$$
\begin{aligned}
G L_{\otimes}\left(\otimes_{i=1}^{l} \mathbb{R}^{d_{i}}\right) \times \mathcal{B}_{\otimes}\left(\otimes_{i=1}^{l} \mathbb{R}^{d_{i}}\right) & \longrightarrow \mathcal{B}_{\otimes}\left(\otimes_{i=1}^{l} \mathbb{R}^{d_{i}}\right) \\
(T, Q) & \mapsto T Q:=\{T u: u \in Q\} .
\end{aligned}
$$

Clearly, it is a continuous action. Indeed, it is the restriction of the natural action of the general linear group $G L\left(\otimes_{i=1}^{l} \mathbb{R}^{d_{i}}\right)$ on the space of 0-symmetric convex bodies in $\otimes_{i=1}^{l} \mathbb{R}^{d_{i}}$, which is continuous ([3, p. 210]).

For simplicity of notation, we usually write $G L_{\otimes}$ and $O_{\otimes}$ instead of $G L_{\otimes}\left(\otimes_{i=1}^{l} \mathbb{R}^{d_{i}}\right)$ and $O_{\otimes}\left(\otimes_{i=1}^{l} \mathbb{R}^{d_{i}}\right)$. Remember that throughout the paper $l \geq 2$ and $d_{i} \geq 2$ are integers.

Theorem 4.1. The action of $G L_{\otimes}\left(\otimes_{i=1}^{l} \mathbb{R}^{d_{i}}\right)$ on $\mathcal{B}_{\otimes}\left(\otimes_{i=1}^{l} \mathbb{R}^{d_{i}}\right)$ is proper.

Proof. First notice that since $G L_{\otimes}$ is a closed subgroup of $G L\left(\otimes_{i=1}^{l} \mathbb{R}^{d_{i}}\right)$, see [15, Proposition 3.11], it is a locally compact Lie group.

Now, let $P \in \mathcal{B}_{\otimes}$ and $\varepsilon>0$ be such that $2 \varepsilon B_{2}^{d_{1}, \ldots, d_{l}} \subseteq P$. We claim that $V_{P}(\varepsilon)$ is a small neighborhood of $P$. To prove this, observe that for each 0 -symmetric convex body $C \subset \otimes_{i=1}^{l} \mathbb{R}^{d_{i}}$, there exists $\lambda>0$ such that $\lambda B_{2}^{d_{1}, \ldots, d_{l}} \subseteq C$. We will show that the transporter $\Gamma=\left\{T \in G L_{\otimes}: T V_{P}(\varepsilon) \cap V_{C}(\lambda) \neq \emptyset\right\}$ has compact closure in $G L_{\otimes}$. To that end, we will prove that every sequence in $\Gamma$ has a convergent subsequence in $G L_{\otimes}$.

Let $T_{k}, k \in \mathbb{N}$, be a sequence contained in $\Gamma$. Then, for every $k \in \mathbb{N}$, there exists $Q_{k} \in V_{P}(\varepsilon)$ such that $T_{k}\left(Q_{k}\right) \in V_{C}(\lambda)$. Thus, by Lemma 3.6, $\varepsilon B_{2}^{d_{1}, \ldots, d_{l}} \subseteq Q_{k}$ for all $k$. Also, since $\delta^{H}\left(T_{k}\left(Q_{k}\right), C\right)<\lambda$, we have that $T_{k}\left(Q_{k}\right) \subseteq C+\lambda B_{2}^{d_{1}, \ldots, d_{l}}$. Hence,

$$
T_{k}\left(\varepsilon B_{2}^{d_{1}, \ldots, d_{l}}\right) \subseteq T_{k}\left(Q_{k}\right) \subseteq C+\lambda B_{2}^{d_{1}, \ldots, d_{l}} .
$$

The latter implies that the sequence $\left\|T_{k}\right\|$ is bounded (here $\left\|T_{k}\right\|$ is the operator norm of $T_{k}$ on $\otimes_{H, i=1}^{l} \mathbb{R}^{d_{i}}$ ). We thus have that $T_{k}$ is a bounded sequence of operators between finite dimensional spaces. Therefore $T_{k}$ must have a convergent subsequence.

Let us denote by $T \in \mathcal{L}\left(\otimes_{H, i=1}^{l} \mathbb{R}^{d_{i}}\right)$ to the limit of such subsequence $T_{k_{i}}$. We will prove that $T$ belongs to $G L_{\otimes}$. Since $G L_{\otimes}$ is a closed subgroup of $G L\left(\otimes_{i=1}^{l} \mathbb{R}^{d_{i}}\right)$, see [15, Proposition 3.11], it is enough to prove that $T$ is a linear isomorphism. To do this, observe that $Q_{k_{i}} \in V_{P}(\varepsilon)$ and $T_{k_{i}}\left(Q_{k_{i}}\right) \in V_{C}(\lambda)$. Thus, from Lemma 3.7 there exists a subsubsequence $Q_{k_{i_{j}}}$ such that $Q_{k_{i_{j}}}$ and $T_{k_{i_{j}}}\left(Q_{k_{i_{j}}}\right)$ converge to some tensorial bodies $Q, D$ respectively. This yields to $T Q=D$ which proves that $T$ is a linear isomorphism. This completes the proof.

Let us recall that $\mathscr{E}_{\otimes}\left(\otimes_{i=1}^{l} \mathbb{R}^{d_{i}}\right)$ consists of the ellipsoids in the class of tensorial bodies, see Section 2.1. The next result extends, to the class of tensorial bodies, Corollary 3.10 of [5] which proves that the space of ellipsoids in $\mathbb{R}^{d}$ is homeomorphic to $\mathbb{R}^{p}$, with $p=\frac{d(d+1)}{2}$.

Corollary 4.2. $\mathscr{E}_{\otimes}\left(\otimes_{i=1}^{l} \mathbb{R}^{d_{i}}\right)$ is homeomorphic to $\mathbb{R}^{p}$ with $p=\frac{d_{1}\left(d_{1}+1\right)}{2}+\cdots+\frac{d_{l}\left(d_{l}+1\right)}{2}$.

Proof. Observe that $O_{\otimes}$ is the estabilizer of $B_{2}^{d_{1}, \ldots, d_{l}}$ thus, by Theorem 4.1 and [29, Proposition 1.1.5], the $G L_{\otimes}$-orbit of $B_{2}^{d_{1}, \ldots, d_{l}}$ is homeomorphic to the quotient $G L_{\otimes} / O_{\otimes}$. The result then follows from [15, Corollary 4.3], which shows that the $G L_{\otimes}$-orbit of $B_{2}^{d_{1}, \ldots, d_{l}}$ is $\mathscr{E} \otimes\left(\otimes_{i=1}^{l} \mathbb{R}^{d_{i}}\right)$, and from Corollary A.5. 


\section{The $G L_{\otimes}$-equivariant retraction $\operatorname{conv}_{\otimes}$}

Given a tensorial body $Q \subset \otimes_{i=1}^{l} \mathbb{R}^{d_{i}}$, we define $c o n v_{\otimes}$ as the map sending $Q$ to the projective tensor product of its associated 0 -symmetric bodies $Q^{i}, i=1, \ldots, l$, see Remark 2.3. That is,

$$
\begin{aligned}
\operatorname{conv}_{\otimes}: \mathcal{B}_{\otimes}\left(\otimes_{i=1}^{l} \mathbb{R}^{d_{i}}\right) & \longrightarrow \mathcal{B}_{\otimes}\left(\otimes_{i=1}^{l} \mathbb{R}^{d_{i}}\right) \\
Q & \mapsto Q^{1} \otimes_{\pi} \cdots \otimes_{\pi} Q^{l} .
\end{aligned}
$$

The set of fixed points of $\operatorname{conv}_{\otimes}$ is $\Pi:=\left\{Q \in \mathcal{B}_{\otimes}\left(\otimes_{i=1}^{l} \mathbb{R}^{d_{i}}\right):\right.$ conv $\left._{\otimes}(Q)=Q\right\}$. Below, we prove that $c o n v_{\otimes}$ is a $G L_{\otimes}$-equivariant retraction from the space of tensorial bodies onto $\Pi$, see (3) in Proposition 4.4.

Lemma 4.3. $\Pi$ is the image of $\mathcal{B}\left(d_{1}\right) \times \cdots \times \mathcal{B}\left(d_{l}\right)$ under the projective tensor product $\otimes_{\pi}$.

Proof. Let us suppose that $Q \in \Pi$, then $Q=Q^{1} \otimes_{\pi} \cdots \otimes_{\pi} Q^{l}$ and it is the image of $\left(Q^{1}, \ldots, Q^{l}\right)$ under $\otimes_{\pi}$. On the other hand, if $Q$ is such that $Q=Q_{1} \otimes_{\pi} \cdots \otimes_{\pi} Q_{l}$ for some 0 -symmetric convex bodies $Q_{i} \subset \mathbb{R}^{d_{i}}$, then $Q \in \mathcal{B}_{Q_{1}, \ldots, Q_{l}} \cap \mathcal{B}_{Q^{1}, \ldots, Q^{l}}$. Therefore, by [15, Proposition 3.6], there exist $\lambda_{i}>0, i=1, \ldots, l$, such that $\lambda_{1} \cdots \lambda_{l}=1$ and $Q^{i}=\lambda_{i} Q_{i}$, for all $i$. From this, $Q^{1} \otimes_{\pi} \cdots \otimes_{\pi} Q^{l}=Q_{1} \otimes_{\pi} \cdots \otimes_{\pi} Q_{l}$ and $Q \in \Pi$, so the proof is completed.

The next proposition collects the basic properties of $\operatorname{conv}_{\otimes}$. It is based on an alternative definition of this map stated in terms of the set of decomposable vectors. We follow the notation of [13. There, this set is denoted by

$$
\Sigma_{\mathbb{R}^{d_{1}}, \ldots, \mathbb{R}^{d_{l}}}:=\left\{x^{1} \otimes \cdots \otimes x^{l}: x^{i} \in \mathbb{R}^{d_{i}}, i=1, \ldots, l\right\} .
$$

Proposition 4.4. The following statements hold:

1. For every tensorial body $Q \subset \otimes_{i=1}^{l} \mathbb{R}^{d_{i}}, \operatorname{conv}_{\otimes}(Q)=\operatorname{conv}\left(Q \cap \Sigma_{\mathbb{R}^{d_{1}}, \ldots, \mathbb{R}^{d_{l}}}\right)$.

2. The map conv $\otimes$ is constant on every $\mathcal{B}_{Q_{1}, \ldots, Q_{l}}\left(\otimes_{i=1}^{l} \mathbb{R}^{d_{i}}\right)$.

3. The map conv ${ }_{\otimes}$ is a $G L_{\otimes}$-equivariant retraction from $\mathcal{B}_{\otimes}\left(\otimes_{i=1}^{l} \mathbb{R}^{d_{i}}\right)$ onto $\Pi$.

Proof. To prove (1) observe that from Theorem 2.2. each tensorial body $Q$ is a tensorial body with respect to $Q^{1}, \ldots, Q^{l}$, so by (2.2), we have $Q^{1} \otimes_{\pi} \cdots \otimes_{\pi} Q^{l} \subseteq \operatorname{conv}\left(Q \cap \Sigma_{\mathbb{R}^{d_{1}}, \ldots, \mathbb{R}^{d_{l}}}\right)$. To show the other inclusion, notice that every $w=x^{1} \otimes \cdots \otimes x^{l} \in Q$ can be written as $w=$ $y^{1} \otimes \cdots \otimes y^{l}$ where $y^{i}=g_{Q^{i}}\left(\frac{x^{i}}{g_{Q^{i}}\left(x^{i}\right)}\right) x^{i}, i=1, \ldots, l-1$, and $y^{l}=g_{Q^{1}}\left(x^{1}\right) \cdots g_{Q^{l-1}}\left(x^{l-1}\right) x^{l}$. Clearly each $y^{i} \in Q^{i}$ for $i=1, \ldots, l-1$ and, from (2.2),$y^{l} \in Q^{l}$. Hence, $w \in Q^{1} \otimes_{\pi} \cdots \otimes_{\pi} Q^{l}$. The desired inclusion then follows by convexity.

(2) We will prove that for every $Q \in \mathcal{B}_{Q_{1}, \ldots, Q_{l}}$,

$$
\operatorname{conv}_{\otimes}(Q)=Q_{1} \otimes_{\pi} \cdots \otimes_{\pi} Q_{l} .
$$

Let $Q \in \mathcal{B}_{Q_{1}, \ldots, Q_{l}}$, then $Q \in \mathcal{B}_{Q_{1}, \ldots, Q_{l}} \cap \mathcal{B}_{Q^{1}, \ldots, Q^{l}}$ and, from [15, Proposition 3.6], there exist $\lambda_{i}>0, i=1, \ldots, l$, such that $\lambda_{1} \cdots \lambda_{l}=1$ and $Q^{i}=\lambda_{i} Q_{i}$, for all $i$. Hence, $Q^{1} \otimes_{\pi} \cdots \otimes_{\pi} Q^{l}=$ $Q_{1} \otimes_{\pi} \cdots \otimes_{\pi} Q_{l}$ and $\operatorname{conv}_{\otimes}(Q)=Q_{1} \otimes_{\pi} \cdots \otimes_{\pi} Q_{l}$, as desired.

(3) To prove the equivariance of $c o n v_{\otimes}$, it is enough to recall that linear maps preserve convex hulls. Hence, $T\left(\operatorname{conv}\left(Q \cap \Sigma_{\mathbb{R}^{d_{1}}, \ldots, \mathbb{R}^{d_{l}}}\right)\right)=\operatorname{conv}\left(T\left(Q \cap \Sigma_{\mathbb{R}^{d_{1}}, \ldots, \mathbb{R}^{d_{l}}}\right)\right)=\operatorname{conv}((T Q) \cap$ 
$\left.\Sigma_{\mathbb{R}^{d_{1}}, \ldots, \mathbb{R}^{d_{l}}}\right)$, for every $T \in G L_{\otimes}$. The latter along with $(1)$ shows that $T\left(\operatorname{conv}_{\otimes}(Q)\right)=$ $c o n v_{\otimes}(T Q)$.

To prove that conv $_{\otimes}$ is continuous, let $Q_{k}, k \in \mathbb{N}$, be a sequence in $\mathcal{B}_{\otimes}$ converging to a tensorial body $Q$. We will see that $\operatorname{conv}_{\otimes}\left(Q_{k}\right)$ converges to $\operatorname{conv}_{\otimes}(Q)$. By $(2)$ in Lemma 3.1. $g_{Q_{k}}$ converges uniformly (on compact sets) to $g_{Q}$. Thus, from [15, Proposition 3.7], $g_{\left(Q_{k}^{i}\right)^{\circ}}$ converges uniformly (on compact sets) to $g_{\left(Q^{i}\right)^{\circ}}$. So, by (13.1),$Q_{k}^{i}$ goes to $Q^{i}$ for each $i=1, \ldots, l$. Hence, by the continuity of $\otimes_{\pi}$ (Proposition [3.3), $Q_{k}^{1} \otimes_{\pi} \cdots \otimes_{\pi} Q_{k}^{l}$ converges to $Q^{1} \otimes_{\pi} \cdots \otimes_{\pi} Q^{l}$ and $c o n v_{\otimes}$ is continuous.

To show that $\operatorname{conv}_{\otimes}$ is a retraction onto $\Pi$, notice that each tensorial body $Q$ is such that $Q, Q^{1} \otimes_{\pi} \cdots \otimes_{\pi} Q^{l} \in \mathcal{B}_{Q^{1}, \ldots, Q^{l}}$, then by (4.1), $\operatorname{conv}_{\otimes}\left(\operatorname{conv}_{\otimes}(Q)\right)=\operatorname{conv}_{\otimes}\left(Q^{1} \otimes_{\pi} \cdots \otimes_{\pi} Q^{l}\right)=$ $Q^{1} \otimes_{\pi} \cdots \otimes_{\pi} Q^{l}=\operatorname{conv}_{\otimes}(Q)$.

\subsection{A global slice for the space of tensorial bodies $\mathcal{B}_{\otimes}\left(\otimes_{i=1}^{l} \mathbb{R}^{d_{i}}\right)$}

Given a 0 -symmetric convex body $Q \subset \mathbb{R}^{d}$, the Löwner ellipsoid $L o ̈ w(Q)$ and John ellipsoid $\operatorname{John}(Q)$ are defined as the ellipsoid of minimal volume (resp. maximal volume) containing $Q$ (resp. contained in $Q$ ). Both ellipsoids are fundamental tools in the study of convex bodies and finite dimensional normed spaces (see for instance [35. Chapter 3]). One of their main features is the uniqueness, which was proved by F. John [19]. This property enables the definition of the Löwner map, Löw, as the map sending each 0-symmetric convex body $Q \subset \mathbb{R}^{d}$ to its Löwner ellipsoid $\operatorname{Löw}(Q)$, see [5, Section 3.2]. Among its fundamental properties, in [5, Theorem 3.6], it is proved that the Löwner map is a $G L(d)$-equivariant retraction onto the set of ellipsoids in $\mathbb{R}^{d}$. By means of this map, we will exhibit a compact $O_{\otimes}$-global slice for the space of tensorial bodies (Theorem 4.7).

The relation between Löwner and John ellipsoids and the tensor products $\otimes_{\pi}, \otimes_{\epsilon}$ is established in [7, Lemma 1] and [14, Proposition 3.14]. There, it is proved that Löwner and John ellipsoids are preserved under the projective $\otimes_{\pi}$ and the injective tensor product $\otimes_{\epsilon}$, respectively. In [7, Lemma 1], it is showed that for every tuple of 0 -symmetric convex bodies $Q_{i} \subset \mathbb{R}^{d_{i}}, i=1, \ldots, l$, the Löwner ellipsoid of the projective tensor product is the Hilbertian tensor product of the Löwner ellipsoids $L \ddot{o w}\left(Q_{i}\right)$. That is,

$$
\operatorname{Löw}\left(Q_{1} \otimes_{\pi} \cdots \otimes_{\pi} Q_{l}\right)=\operatorname{Lö} w\left(Q_{1}\right) \otimes_{2} \cdots \otimes_{2} \operatorname{Lö} w\left(Q_{l}\right) .
$$

As a consequence of (4.2), Löwner ellipsoids of projective tensor products of 0-symmetric convex bodies are tensorial ellipsoids. This allows us to define the map $l_{\otimes}$ as the composition of two retractions $l_{\otimes}:=L \ddot{o} w \circ \operatorname{conv}_{\otimes}$,

$$
\begin{aligned}
l_{\otimes}: \mathcal{B}_{\otimes}\left(\otimes_{i=1}^{l} \mathbb{R}^{d_{i}}\right) & \rightarrow \mathscr{E}_{\otimes}\left(\otimes_{i=1}^{l} \mathbb{R}^{d_{i}}\right) \\
Q & \mapsto L \ddot{o} w\left(Q^{1} \otimes_{\pi} \cdots \otimes_{\pi} Q^{l}\right) .
\end{aligned}
$$

Below we prove that $l_{\otimes}$ is a $G L_{\otimes}$-equivariant retraction onto the space of tensorial ellipsoids (Proposition 4.5). This map will be used to show that the subset $\mathscr{L}_{\otimes}\left(\otimes_{i=1}^{l} \mathbb{R}^{d_{i}}\right)$ which consists of the tensorial bodies $Q \subset \otimes_{i=1}^{l} \mathbb{R}^{d_{i}}$ such that $l_{\otimes}(Q)=B_{2}^{d_{1}, \ldots, d_{l}}$ is a compact global $O_{\otimes}$-slice for the space of tensorial bodies $\mathcal{B}_{\otimes}\left(\otimes_{i=1}^{l} \mathbb{R}^{d_{i}}\right)$, see Theorem 4.7. To simplify notation, we will write $\mathscr{E}_{\otimes}$ and $\mathscr{L}_{\otimes}$ instead of $\mathscr{E}_{\otimes}\left(\otimes_{i=1}^{l} \mathbb{R}^{d_{i}}\right)$ and $\mathscr{L}_{\otimes}\left(\otimes_{i=1}^{l} \mathbb{R}^{d_{i}}\right)$.

Proposition 4.5. The map $l_{\otimes}$ is a $G L_{\otimes}$-equivariant retraction from $\mathcal{B}_{\otimes}\left(\otimes_{i=1}^{l} \mathbb{R}^{d_{i}}\right)$ onto $\mathscr{E} \otimes\left(\otimes_{i=1}^{l} \mathbb{R}^{d_{i}}\right)$. 
Proof. The map $l_{\otimes}$ is continuous and $G L_{\otimes}$-equivariant due to the Löwner map $L \ddot{o w}$ is a continuous $G L\left(\otimes_{i=1}^{l} \mathbb{R}^{d_{i}}\right)$-equivariant map [5, Theorem 3.6], and the map conv $v_{\otimes}$ is continuous and $G L_{\otimes}$-equivariant (see Proposition 4.4). To prove that $l_{\otimes}$ is a retraction. Let $\mathcal{E} \subset \otimes_{i=1}^{l} \mathbb{R}^{d_{i}}$ be a tensorial ellipsoid, then, by [15, Corollary 4.3], there exist $T_{i} \in G L\left(d_{i}\right)$, $i=1, \ldots, l$, such that $\mathcal{E}=T_{1} \otimes \cdots \otimes T_{l}\left(B_{2}^{d_{1}, \ldots, d_{l}}\right)$. Thus,

$$
\begin{gathered}
l_{\otimes}(\mathcal{E})=l_{\otimes}\left(T_{1} \otimes \cdots \otimes T_{l}\left(B_{2}^{d_{1}, \ldots, d_{l}}\right)\right)=\left(T_{1} \otimes \cdots \otimes T_{l}\right) l_{\otimes}\left(B_{2}^{d_{1}, \ldots, d_{l}}\right) \\
=T_{1} \otimes \cdots \otimes T_{l}\left(B_{2}^{d_{1}, \ldots, d_{l}}\right)=\mathcal{E} .
\end{gathered}
$$

The third equality follows from (2.9), (4.1) and (4.2).

Proposition 4.6. $\mathscr{L}_{\otimes}\left(\otimes_{i=1}^{l} \mathbb{R}^{d_{i}}\right)$ enjoys of the following properties:

1. $\mathscr{L}_{\otimes}\left(\otimes_{i=1}^{l} \mathbb{R}^{d_{i}}\right)$ is $O_{\otimes}$-invariant.

2. The $G L_{\otimes}$-saturation of $\mathscr{L}_{\otimes}\left(\otimes_{i=1}^{l} \mathbb{R}^{d_{i}}\right)$ coincides with $\mathcal{B}_{\otimes}\left(\otimes_{i=1}^{l} \mathbb{R}^{d_{i}}\right)$.

3. Let $T \in G L_{\otimes}\left(\otimes_{i=1}^{l} \mathbb{R}^{d_{i}}\right)$. If $T\left(\mathscr{L}_{\otimes}\left(\otimes_{i=1}^{l} \mathbb{R}^{d_{i}}\right)\right) \cap \mathscr{L}_{\otimes}\left(\otimes_{i=1}^{l} \mathbb{R}^{d_{i}}\right) \neq \emptyset$, then $T$ is an orthogonal map.

4. $\mathscr{L}_{\otimes}\left(\otimes_{i=1}^{l} \mathbb{R}^{d_{i}}\right)$ is compact.

Proof. (1). Let $Q \in \mathscr{L}_{\otimes}$ and let $U$ be an orthogonal map in $O_{\otimes}$. By the equivariance of $l_{\otimes}$ (Proposition 4.5),$l_{\otimes}(U Q)=U\left(l_{\otimes}(Q)\right)=U\left(B_{2}^{d_{1}, \ldots, d_{l}}\right)=B_{2}^{d_{1}, \ldots, d_{l}}$. Therefore $U(Q) \in \mathscr{L}_{\otimes}$ and the invariance of $\mathscr{L}_{\otimes}$ is proved.

(2). Let $Q \subset \otimes_{i=1}^{l} \mathbb{R}^{d_{i}}$ be an arbitrary tensorial body, we will see that it belongs to the $G L_{\otimes}$-saturation of $\mathscr{L}_{\otimes}$. Since for each $i=1, \ldots, l$, there exists $T_{i} \in G L\left(d_{i}\right)$ such that $L \ddot{o} w\left(Q^{i}\right)=T_{i}\left(B_{2}^{d_{i}}\right)$, then, by setting $P:=T_{1}^{-1} \otimes \cdots \otimes T_{l}^{-1}(Q)$, we have:

$$
\begin{gathered}
l_{\otimes}(P)=l_{\otimes}\left(T_{1}^{-1} \otimes \cdots \otimes T_{l}^{-1}(Q)\right) \stackrel{*}{=} T_{1}^{-1} \otimes \cdots \otimes T_{l}^{-1}\left(l_{\otimes}(Q)\right) \\
\stackrel{* *}{=} T_{1}^{-1} \otimes \cdots \otimes T_{l}^{-1}\left(\operatorname{Lö} w\left(Q^{1}\right) \otimes_{2} \cdots \otimes_{2} \operatorname{Löw}\left(Q^{l}\right)\right) \\
=T_{1}^{-1} \otimes \cdots \otimes T_{l}^{-1}\left(T_{1}\left(B_{2}^{d_{1}}\right) \otimes_{2} \cdots \otimes_{2} T_{l}\left(B_{2}^{d_{l}}\right)\right) \\
\stackrel{* * *}{=} B_{2}^{d_{1}} \otimes_{2} \cdots \otimes_{2} B_{2}^{d_{l}}=B_{2}^{d_{1}, \ldots, d_{l}} .
\end{gathered}
$$

(*) follows from Proposition 4.5. (**) follows from (4.2). (***) follows from the second equality in (2.10).

Therefore $P \in \mathscr{L}_{\otimes}$ and $Q=T_{1} \otimes \cdots \otimes T_{l}(P)$ belongs to the $G L_{\otimes}$-saturation of $\mathscr{L}_{\otimes}$.

(3). Let $T \in G l_{\otimes}$ such that $T Q \in \mathscr{L}_{\otimes}$, for some $Q \in \mathscr{L}_{\otimes}$. Then, by the $G L_{\otimes}$ equivariance of $l_{\otimes}, T\left(B_{2}^{d_{1} \ldots, d_{l}}\right)=T\left(l_{\otimes}(Q)\right)=l_{\otimes}(T Q)=B_{2}^{d_{1} \ldots, d_{l}}$. This proves that $T$ is an orthogonal map in $G L_{\otimes}$. So, $T \in O_{\otimes}$.

(4). We will prove that any sequence in $\mathscr{L}_{\otimes}$ has a convergent subsequence. Let $Q_{k}$ be a sequence contained in $\mathscr{L}_{\otimes}$. Then for each $k, \operatorname{Lö} w\left(Q_{k}^{1} \otimes_{\pi} \cdots \otimes_{\pi} Q_{k}^{l}\right)=B_{2}^{d_{1}, \ldots, d_{l}}$ and so $Q_{k}^{1} \otimes_{\pi} \cdots \otimes_{\pi} Q_{k}^{l}$ belongs to the compact set $\mathscr{L}\left(\otimes_{i=1}^{l} \mathbb{R}^{d_{i}}\right) \subset \mathcal{B}\left(\otimes_{i=1}^{l} \mathbb{R}^{d_{i}}\right)$ of [3], Remark 1]. Consequently, there exists a subsequence $Q_{k_{j}}^{1} \otimes_{\pi} \cdots \otimes_{\pi} Q_{k_{j}}^{l}$ converging to some 0 -symmetric convex body $D \subset \otimes_{i=1}^{l} \mathbb{R}^{d_{i}}$, such that $L \ddot{o} w(D)=B_{2}^{d_{1}, \ldots, d_{l}}$. Hence, from (2) in Lemma 3.1 and [15, Proposition 3.7], we have that $D$ is a tensorial body for which $Q_{k_{j}}^{i}$ and $\left(Q_{k_{j}}^{i}\right)^{\circ}$ converge to $D^{i}$ and $\left(D^{i}\right)^{\circ}$ respectively. 
Now, by the continuity of $\otimes_{\pi}$ and $\otimes_{\epsilon}$ (Proposition 3.3), we have that $Q_{k_{j}}^{1} \otimes_{\pi} \cdots \otimes_{\pi} Q_{k_{j}}^{l}$ and $Q_{k_{j}}^{1} \otimes_{\epsilon} \cdots \otimes_{\epsilon} Q_{k_{j}}^{l}$ converge to $D^{1} \otimes_{\pi} \cdots \otimes_{\pi} D^{l}$ and $D^{1} \otimes_{\epsilon} \cdots \otimes_{\epsilon} D^{l}$, respectively. Since we also have that $Q_{k_{j}}^{1} \otimes_{\pi} \cdots \otimes_{\pi} Q_{k_{j}}^{l} \subseteq Q_{k_{j}} \subseteq Q_{k_{j}}^{1} \otimes_{\epsilon} \cdots \otimes_{\epsilon} Q_{k_{j}}^{l}$, then by the Blaschke selection theorem ([32, Theorem 1.8.6]), we can suppose that $Q_{k_{j}}$ converges to some compact convex set $Q$. In such case, we must have that $D^{1} \otimes_{\pi} \cdots \otimes_{\pi} D^{l} \subseteq Q \subseteq D^{1} \otimes_{\epsilon} \cdots \otimes_{\epsilon} D^{l}$ and so $Q$ is a tensorial body. Finally, from the continuity of $l_{\otimes}$ (Proposition 4.5), it follows that $l_{\otimes}(Q)=B_{2}^{d_{1}, \ldots, d_{l}}$. This shows that $Q \in \mathscr{L}_{\otimes}$ and the proof is completed.

Theorem 4.7. $\mathscr{L}_{\otimes}\left(\otimes_{i=1}^{l} \mathbb{R}^{d_{i}}\right)$ is a compact global $O_{\otimes}$-slice for the proper $G L_{\otimes}$-space $\mathcal{B}_{\otimes}\left(\otimes_{i=1}^{l} \mathbb{R}^{d_{i}}\right)$.

Proof. The compactness of $\mathscr{L}_{\otimes}$ was proved in Proposition 4.6. Now, we will prove that it is a global $G L_{\otimes}$-slice for the space of tensorial bodies. Since $\mathscr{E}_{\otimes}$ is the $G L_{\otimes}$-orbit of $B_{2}^{d_{1} \ldots, d_{l}}$, see [15, Corollary 4.3], and $O_{\otimes}$ is the stabilizer of $B_{2}^{d_{1} \ldots, d_{l}}$, then, by [29, Proposition 1.1.5], there is a $G L_{\otimes}$-equivariant homeomorphism between $\mathscr{E} \otimes$ and $G L_{\otimes} / O_{\otimes}$. The latter together with Proposition 4.5 and the fact that $\mathscr{L}_{\otimes}\left(\otimes_{i=1}^{l} \mathbb{R}^{d_{i}}\right)=l_{\otimes}^{-1}\left(B_{2}^{d_{1} \ldots, d_{l}}\right)$ yield a $G L_{\otimes^{-}}$ equivariant map $f: \mathcal{B}_{\otimes} \rightarrow G L_{\otimes} / O_{\otimes}$ such that $\mathscr{L}_{\otimes}\left(\otimes_{i=1}^{l} \mathbb{R}^{d_{i}}\right)=f^{-1}\left(O_{\otimes}\left(\otimes_{i=1}^{l} \mathbb{R}^{d_{i}}\right)\right)$.

\subsection{Topological representatives for $\mathcal{B}_{\otimes}\left(\otimes_{i=1}^{l} \mathbb{R}^{d_{i}}\right)$ and $\mathcal{B} \mathcal{M}_{\otimes}\left(\otimes_{i=1}^{l} \mathbb{R}^{d_{i}}\right)$}

Here, we exhibit topological representatives for both the space of tensorial bodies and the compactum $\mathcal{B M}_{\otimes}\left(\otimes_{i=1}^{l} \mathbb{R}^{d_{i}}\right)$. Namely, in Corollary 4.9, we prove that $\mathcal{B} \mathcal{M}_{\otimes}\left(\otimes_{i=1}^{l} \mathbb{R}^{d_{i}}\right)$ is homeomorphic to two orbit spaces $\mathcal{B}_{\otimes} / G L_{\otimes}$ and $\mathscr{L}_{\otimes} / O_{\otimes}$. In Corollaries 4.10 and 4.11, we prove that $\mathcal{B}_{\otimes}$ is homeomorphic to the product $\mathscr{L}_{\otimes} \times \mathscr{E} \otimes$ and $\mathscr{L}_{\otimes} \times \mathbb{R}^{p}$, respectively. It is worth to notice that these results extend, to the context of tensorial bodies, Corollary 1 of [3] and Corollaries 3.8 and 3.9 of [5] about the Banach-Mazur compactum and the space of convex bodies in $\mathbb{R}^{d}$, respectively.

Proposition 4.8. The following statements hold:

1. The orbit space $\mathcal{B}_{\otimes}\left(\otimes_{i=1}^{l} \mathbb{R}^{d_{i}}\right) / G L_{\otimes}\left(\otimes_{i=1}^{l} \mathbb{R}^{d_{i}}\right)$ is compact.

2. The spaces $\mathscr{L}_{\otimes}\left(\otimes_{i=1}^{l} \mathbb{R}^{d_{i}}\right) / O_{\otimes}\left(\otimes_{i=1}^{l} \mathbb{R}^{d_{i}}\right)$ and $\mathcal{B}_{\otimes}\left(\otimes_{i=1}^{l} \mathbb{R}^{d_{i}}\right) / G L_{\otimes}\left(\otimes_{i=1}^{l} \mathbb{R}^{d_{i}}\right)$ are homeomorphic.

Proof. (1). From Proposition [4.6, $\mathscr{L}_{\otimes}$ is compact and $G L_{\otimes}\left(\mathscr{L}_{\otimes}\right)=\mathcal{B}_{\otimes}$. Thus, by the continuity of the orbit map $\pi: \mathcal{B}_{\otimes} \rightarrow \mathcal{B}_{\otimes} / G L_{\otimes}$, we have that $\pi\left(\mathscr{L}_{\otimes}\right)=\mathcal{B}_{\otimes} / G L_{\otimes}$ is compact.

(2). Denote by $\pi_{\mid}$the restriction of the orbit map to $\mathscr{L}_{\otimes}$. From (1), we know that $\pi_{\mid}$is a continuous surjective map from $\mathscr{L}_{\otimes}\left(\otimes_{i=1}^{l} \mathbb{R}^{d_{i}}\right)$ onto $\mathcal{B}_{\otimes} / G L_{\otimes}$. Also, notice that from (3) in Proposition 4.6, for every $P, Q \in \mathscr{L}_{\otimes}$ we have that $\pi_{\mid}(P)=\pi_{\mid}(Q)$ if and only if $P, Q$ have the same $O_{\otimes}$-orbit. From this, $\pi_{\mid}$induces a continuous bijective map $\rho: \mathscr{L}_{\otimes} / O_{\otimes} \rightarrow \mathcal{B}_{\otimes} / G L_{\otimes}$. Since $\mathscr{L}_{\otimes}$ is compact (Proposition 4.6), the same holds for $\mathscr{L}_{\otimes} / O_{\otimes}$, see [9, Theorem 3.1]. Finally, due to $\mathcal{B}_{\otimes} / G L_{\otimes}$ is Hausdorff (see [29, Proposition 1.1.4]), we have that $\rho$ is a homeomorphism between $\mathscr{L}_{\otimes} / O_{\otimes}$ and $\mathcal{B}_{\otimes} / G L_{\otimes}$. This completes the proof.

Recall that $\mathcal{B} \mathcal{M}_{\otimes}\left(\otimes_{i=1}^{l} \mathbb{R}^{d_{i}}\right)$ endowed with the metric $\log \delta_{\otimes}^{B M}$ is a compact metric space ([15, Theorem 3.13]). It consists of the equivalence classes of tensorial bodies determined by the relation $P \sim Q$ if and only if $T(P)=Q$ for some $T \in G L_{\otimes}$, see (2.8). 
Corollary 4.9. $\mathcal{B} \mathcal{M}_{\otimes}\left(\otimes_{i=1}^{l} \mathbb{R}^{d_{i}}\right)$ is homeomorphic to $\mathcal{B}_{\otimes}\left(\otimes_{i=1}^{l} \mathbb{R}^{d_{i}}\right) / G L_{\otimes}\left(\otimes_{i=1}^{l} \mathbb{R}^{d_{i}}\right)$ and $\mathscr{L}_{\otimes}\left(\otimes_{i=1}^{l} \mathbb{R}^{d_{i}}\right) / O_{\otimes}\left(\otimes_{i=1}^{l} \mathbb{R}^{d_{i}}\right)$.

Proof. Let $\Psi: \mathcal{B}_{\otimes}\left(\otimes_{i=1}^{l} \mathbb{R}^{d_{i}}\right) \rightarrow \mathcal{B} \mathcal{M}_{\otimes}\left(\otimes_{i=1}^{l} \mathbb{R}^{d_{i}}\right)$ be the map sending each tensorial body $Q$ to its class $[Q] \in \mathcal{B} \mathcal{M}_{\otimes}$. It is not difficult to prove that, as a consequence of Lemma 3.1 and [15, Proposition 3.7], $\Psi$ is continuous. Clearly it is surjective, and $\Psi(P)=\Psi(Q)$ if and only if $Q$ belongs to the $G L_{\otimes}$-orbit of $P$. Therefore, by the compactness of $\mathcal{B}_{\otimes} / G L_{\otimes}$ (see (1) in Proposition 4.8) and the fact that $\mathcal{B M}_{\otimes}$ is a Hausdorff space, it follows that $\Psi$ induces a homeomorphism between $\mathcal{B}_{\otimes} / G L_{\otimes}$ and $\mathcal{B} \mathcal{M}_{\otimes}$. This proves the first part of the corollary. The second part follows directly from (2) in Proposition 4.8

Corollary 4.10. The following statements hold:

1. There exists an $O_{\otimes}$-equivariant retraction $r: \mathcal{B}_{\otimes}\left(\otimes_{i=1}^{l} \mathbb{R}^{d_{i}}\right) \rightarrow \mathscr{L}_{\otimes}\left(\otimes_{i=1}^{l} \mathbb{R}^{d_{i}}\right)$ such that $r(P)$ belongs to the $G L_{\otimes}$-orbit of $P$.

2. $\mathcal{B}_{\otimes}\left(\otimes_{i=1}^{l} \mathbb{R}^{d_{i}}\right)$ is homeomorphic to $\mathscr{L}_{\otimes}\left(\otimes_{i=1}^{l} \mathbb{R}^{d_{i}}\right) \times \mathscr{E} \otimes\left(\otimes_{i=1}^{l} \mathbb{R}^{d_{i}}\right)$.

Proof. In order to prove the first part of the theorem, we will use Lemma A.6. There, it is proved that $G L_{\otimes}$ is homeomorphic to the product $\mathcal{A} \times O_{\otimes}$ where $\mathcal{A} \subset G L_{\otimes}$ consists of the tensor products $S_{1} \otimes \cdots \otimes S_{l}$ of strictly positive maps $S_{i} \in G L\left(d_{i}\right)$, and the homeomorphism is given by the composition map.

(1) To define the so called retraction, let us first consider $f: G L_{\otimes} \rightarrow \mathscr{E}_{\otimes}$ defined as $f(T):=T\left(B_{2}^{d_{1}, \ldots, d_{l}}\right)$. By [29, Proposition 1.1.5] and [15, Corollary 4.3], $f$ induces a $G L_{\otimes}$-equivariant homeomorphism $\tilde{f}: G L_{\otimes} / O_{\otimes} \rightarrow \mathscr{E}_{\otimes}$. Indeed, $f$ is the composition of the maps:

$$
G L_{\otimes} \stackrel{\pi}{\rightarrow} G l_{\otimes} / O_{\otimes} \stackrel{\tilde{f}}{\rightarrow} \mathscr{E} \otimes
$$

where $\pi$ is the natural quotient map. From the compactness of $O_{\otimes}$, it follows that $\pi$ is closed ([9, Theorem 3.1]). Also, since $f$ is the composition of two closed maps, it must be closed too. From this and Lemma A.6, we know that the restriction $f_{\mid \mathcal{A}}$ is a homeomorphism between $\mathcal{A}$ and $\mathscr{E} \otimes$. Furthermore, if we let $O_{\otimes}$ acts on $\mathcal{A}$ by sending the pair $(U, S) \in O_{\otimes} \times \mathcal{A}$ to $U S U^{-1}$, and on $\mathscr{E}_{\otimes}$ by the action induced from $\mathcal{B}_{\otimes}$, then $f_{\mid \mathcal{A}}$ is an $O_{\otimes}$-equivariant homeomorphism.

Denote by $\xi: \mathscr{E}_{\otimes} \rightarrow \mathcal{A}$ the inverse map of $f_{\mid \mathcal{A}}$, then

$$
[\xi(\mathcal{E})]^{-1} \mathcal{E}=B_{2}^{d_{1}, \ldots, d_{l}} \text { for all } \mathcal{E} \in \mathscr{E}_{\otimes}
$$

We claim that the map $r: \mathcal{B}_{\otimes} \rightarrow \mathscr{L}_{\otimes}$, defined as, $r(Q):=\left[\xi\left(l_{\otimes}(Q)\right)\right]^{-1} Q$ is the desired $O_{\otimes}$-equivariant retraction. By its definition $r$ is continuous and $r(Q)$ belongs to the $G L_{\otimes^{-}}$ orbit of $Q \in \mathcal{B}_{\otimes}$. Also, from (4.3) and the equivariance of $l_{\otimes}$ (Proposition 4.5), we have

$$
l_{\otimes}(r(Q))=l_{\otimes}\left(\left[\xi\left(l_{\otimes}(Q)\right)\right]^{-1} Q\right)=\left[\xi\left(l_{\otimes}(Q)\right)\right]^{-1} l_{\otimes}(Q)=B_{2}^{d_{1}, \ldots, d_{l}} .
$$

This shows that $r(Q) \in \mathscr{L}_{\otimes}$ for all $Q \in \mathcal{B}_{\otimes}$. To prove that it is a retraction onto $\mathscr{L}_{\otimes}$, observe that for every $Q \in \mathscr{L}_{\otimes}$,

$$
r(Q)=\left[\xi\left(l_{\otimes}(Q)\right)\right]^{-1} Q=\left[\xi\left(B_{2}^{d_{1}, \ldots, d_{l}}\right)\right]^{-1} Q=I_{\otimes_{i=1}^{l} \mathbb{R}^{d_{i}}}(Q)=Q .
$$


To prove that $r$ is $O_{\otimes}$-equivariant, let $U \in O_{\otimes}$ and $Q \in \mathcal{B}_{\otimes}$ then

$$
r(U Q)=\left[\xi\left(l_{\otimes}(U Q)\right)\right]^{-1} U Q=\left[\xi\left(U l_{\otimes}(Q)\right)\right]^{-1} U Q .
$$

By the equivariance of $\xi$, we have $\xi\left(U l_{\otimes}(Q)\right)=U \xi\left(l_{\otimes}(Q)\right) U^{-1}$. Thus $\left[\xi\left(U l_{\otimes}(Q)\right)\right]^{-1}=$ $U\left[\xi\left(l_{\otimes}(Q)\right)\right]^{-1} U^{-1}$. Consequently,

$$
r(U Q)=\left(U\left[\xi\left(l_{\otimes}(Q)\right)\right]^{-1} U^{-1}\right) U Q=U\left(\left[\xi\left(l_{\otimes}(Q)\right)\right]^{-1} Q\right)=U(r(Q))
$$

as required.

To prove (2), define $\varphi: \mathcal{B}_{\otimes} \rightarrow \mathscr{L}_{\otimes} \times \mathscr{E}_{\otimes}$ as $\varphi(Q):=\left(r(Q), l_{\otimes}(Q)\right)$, then $\varphi$ is an $O_{\otimes}$-equivariant homeomorphism with inverse map given by $\varphi^{-1}(Q, \mathcal{E})=\xi(\mathcal{E}) Q$.

The next corollary follows directly from the above and Corollary 4.2

Corollary 4.11. $\mathcal{B}_{\otimes}\left(\otimes_{i=1}^{l} \mathbb{R}^{d_{i}}\right)$ is homeomorphic to $\mathscr{L}_{\otimes}\left(\otimes_{i=1}^{l} \mathbb{R}^{d_{i}}\right) \times \mathbb{R}^{p}$, with $p=\frac{d_{1}\left(d_{1}+1\right)}{2}+$ $\cdots+\frac{d_{l}\left(d_{l}+1\right)}{2}$.

\section{A The Lie group structure of $G L_{\otimes}\left(\otimes_{i=1}^{l} \mathbb{R}^{d_{i}}\right)$}

Proposition 3.11 of [15] shows that $G L_{\otimes}\left(\otimes_{i=1}^{l} \mathbb{R}^{d_{i}}\right)$ is a closed subgroup of $G L\left(\otimes_{i=1}^{l} \mathbb{R}^{d_{i}}\right)$, with respect to the topology induced by the operator norm on $\mathcal{L}\left(\otimes_{H, i=1}^{l} \mathbb{R}^{d_{i}}\right)$. Consequently, $G L_{\otimes}\left(\otimes_{i=1}^{l} \mathbb{R}^{d_{i}}\right)$ is a Lie group and $O_{\otimes}\left(\otimes_{i=1}^{l} \mathbb{R}^{d_{i}}\right)$ is a compact subgroup of it.

Given a permutation $\sigma$ on $\{1, \ldots, l\}$ for which $x^{\sigma(1)} \otimes \cdots \otimes x^{\sigma(l)} \in \otimes_{i=1}^{l} \mathbb{R}^{d_{i}}$, whenever $x^{i} \in \mathbb{R}^{d_{i}}, i=1, \ldots, l$, we define the map $U_{\sigma}$ in decomposable vectors as:

$$
U_{\sigma}\left(x^{1} \otimes \cdots \otimes x^{l}\right)=x^{\sigma(1)} \otimes \cdots \otimes x^{\sigma(l)},
$$

and extend it to $\otimes_{i=1}^{l} \mathbb{R}^{d_{i}}$ by multilinearity. The map $U_{\sigma}$ is orthogonal. Indeed, notice that the canonical basis $e_{k_{i}}^{d_{i}}, k_{i}=1, \ldots, d_{i}$, of $\mathbb{R}^{d_{i}}$ is such that $U_{\sigma}\left(e_{k_{1}}^{d_{1}} \otimes \cdots \otimes e_{k_{l}}^{d_{l}}\right)=$ $e_{k_{\sigma(1)}}^{d_{\sigma(1)}} \otimes \cdots \otimes e_{k_{\sigma(l)}}^{d_{\sigma(l)}}$. We will denote by $\mathcal{P}$ to the subset of $G L_{\otimes}\left(\otimes_{i=1}^{l} \mathbb{R}^{d_{i}}\right)$ of orthogonal maps $U_{\sigma}$ as above.

Lemma A.1. The following hold:

1. $\mathcal{P}$ is a finite subgroup of $O_{\otimes}\left(\otimes_{i=1}^{l} \mathbb{R}^{d_{i}}\right)$.

2. Every $T \in G L_{\otimes}\left(\otimes_{i=1}^{l} \mathbb{R}^{d_{i}}\right)$ can be written as $T=\left(T_{1} \otimes \cdots \otimes T_{l}\right) U_{\sigma}$, for some $T_{i} \in$ $G L\left(d_{i}\right), i=1, \ldots, l$, and $U_{\sigma} \in \mathcal{P}$.

Proof. (1) Clearly $\mathcal{P}$ is a finite subset of $O_{\otimes}$ and the identity map on $\otimes_{i=1}^{l} \mathbb{R}^{d_{i}}$ belongs to it. To prove that it is a subgroup, let $U_{\sigma}, U_{\beta} \in \mathcal{P}$, then it can be directly checked that $U_{\sigma}^{-1}=U_{\sigma^{-1}}$ and $U_{\sigma} U_{\beta}=U_{\beta \sigma}$.

(2) Let $T \in G L_{\otimes}$ then, by [25. Corollary 2.1.4], there exist a permutation $\sigma$ on $\{1, \ldots, l\}$ and $T_{i} \in G L\left(d_{i}\right), i=1, \ldots, l$, such that $T\left(x^{1} \otimes \cdots \otimes x^{l}\right)=T_{1}\left(x^{\sigma(1)}\right) \otimes \cdots \otimes T_{l}\left(x^{\sigma(l)}\right)$. Hence, $T=\left(T_{1} \otimes \cdots \otimes T_{l}\right) U_{\sigma}$ as desired.

Proposition A.2. $O_{\otimes}\left(\otimes_{i=1}^{l} \mathbb{R}^{d_{i}}\right)$ is a maximal compact subgroup of $G L_{\otimes}\left(\otimes_{i=1}^{l} \mathbb{R}^{d_{i}}\right)$. 
Proof. Let $K \subset G L_{\otimes}$ be a compact subgroup such that $O_{\otimes} \subseteq K$. We will prove that $K=O_{\otimes}$.

Suppose that $T \in K$ then, by Lemma A.1, there exist $T_{i} \in G L\left(d_{i}\right), i=1, \ldots, l$, and $U_{\sigma} \in \mathcal{P}$ such that $T=T_{1} \otimes \cdots \otimes T_{l} U_{\sigma}$. By the polar decomposition (21, Theorem 60]), each $T_{i}$ can be written as $T_{i}=S_{i} U_{i}$, for some positive linear map $S_{i} \in G L\left(d_{i}\right)$ and $U_{i} \in O\left(d_{i}\right)$. Therefore, $T=S_{1} \otimes \cdots \otimes S_{l} U_{1} \otimes \cdots \otimes U_{l} U_{\sigma}$ and so $S_{1} \otimes \cdots \otimes S_{l}$ is a positive self-adjoint linear map in $K$. Now, from the compactness of $K$, for each eigenvalue $\lambda_{i}$ of $S_{i}$ with unitary eigenvector $z^{i}$ and each integer $n$, the sequence $\left(S_{1} \otimes \cdots \otimes S_{l}\right)^{n}\left(z^{1} \otimes \cdots \otimes z^{l}\right)=$ $\left(\lambda_{1} \cdots \lambda_{l}\right)^{n} z^{1} \otimes \cdots \otimes z^{l}$ is bounded in $\otimes_{i=1}^{l} \mathbb{R}^{d_{i}}$. Since this is only possible if $\lambda_{1} \cdots \lambda_{l}=1$, and this holds for every eigenvalue $\lambda_{1} \cdots \lambda_{l}$ of $S_{1} \otimes \cdots \otimes S_{l}$, we have that $S_{1} \otimes \cdots \otimes S_{l}$ is the identity on $\otimes_{i=1}^{l} \mathbb{R}^{d_{i}}$. Thus, $T \in O_{\otimes}$ and $K \subseteq O_{\otimes}$ as required.

Let us denote by $\otimes\left(G L\left(d_{1}\right), \ldots, G L\left(d_{l}\right)\right)$ the set of tensor products $T_{1} \otimes \cdots \otimes T_{l}$ of linear maps $T_{i} \in G L\left(d_{i}\right)$. Similarly, $\otimes\left(O\left(d_{1}\right), \ldots, O\left(d_{l}\right)\right)$ denotes the set of tensor products $U_{1} \otimes \cdots \otimes U_{l}$ of orthogonal maps $U_{i} \in O\left(d_{i}\right)$. Below, we show that $\otimes\left(G L\left(d_{1}\right), \ldots, G L\left(d_{l}\right)\right)$ and $\otimes\left(O\left(d_{1}\right), \ldots, O\left(d_{l}\right)\right)$ are Lie groups of dimensions $d_{1}^{2}+\cdots+d_{l}^{2}-(l-1)$ and $\frac{d_{1}\left(d_{1}-1\right)}{2}+$ $\cdots+\frac{d_{1}\left(d_{1}-1\right)}{2}-(l-1)$, respectively.

Lemma A.3. Let $N \subset G L\left(d_{1}\right) \times \cdots \times G L\left(d_{l}\right)$ be defined as $N:=\left\{\left(\lambda_{1} I_{d_{1}}, \ldots, \lambda_{l} I_{d_{l}}\right)\right.$ : $\left.\lambda_{1} \cdots \lambda_{l}=1\right\}$. Then $N$ is a normal subgroup and:

1. $\otimes\left(G L\left(d_{1}\right), \ldots, G L\left(d_{l}\right)\right)$ is isomorphic as a Lie group to $G L\left(d_{1}\right) \times \cdots \times G L\left(d_{l}\right) / N$.

2. $\otimes\left(O\left(d_{1}\right), \ldots, O\left(d_{l}\right)\right)$ is isomorphic as a Lie group to $O\left(d_{1}\right) \times \cdots \times O\left(d_{l}\right) / N$.

Proof. We begin by proving that both $\otimes\left(G L\left(d_{1}\right), \ldots, G L\left(d_{l}\right)\right)$ and $\otimes\left(O\left(d_{1}\right), \ldots, O\left(d_{l}\right)\right)$ are closed subgroups of $G L_{\otimes}\left(\otimes_{i=1}^{l} \mathbb{R}^{d_{i}}\right)$ and, in consequence, they are Lie groups. From the properties of the tensor product of linear maps, it follows easily that both of them are subgroups. The closedness follows from two facts. First, they are subsets of the set of decomposable vectors in $\mathcal{L}\left(\mathbb{R}^{d_{1}}, \mathbb{R}^{d_{1}}\right) \otimes \cdots \otimes \mathcal{L}\left(\mathbb{R}^{d_{l}}, \mathbb{R}^{d_{l}}\right)$, which is closed with respect to any norm topology on the tensor space (see [10, Proposition 4.2]). Second, $G L_{\otimes}$ is closed in $G L\left(\otimes_{i=1}^{l} \mathbb{R}^{d_{i}}\right)$, see [15, Proposition 3.11].

We now construct the desired isomorphisms. Let $\Phi: G L\left(d_{1}\right) \times \cdots \times G L\left(d_{l}\right) \rightarrow$ $\otimes\left(G L\left(d_{1}\right), \ldots, G L\left(d_{l}\right)\right)$ be the map sending each tuple $\left(T_{1}, \ldots, T_{l}\right)$ to its tensor product $T_{1} \otimes \cdots \otimes T_{l}$. Also, let $\Phi_{\mid}$the restriction of $\Phi$ to $O\left(d_{1}\right) \times \cdots \times O\left(d_{l}\right)$. Clearly, $\Phi$ and $\Phi$ are surjective maps. Indeed, it is not difficult to prove that they are smooth homomorphisms with Kernel $N$. Thus, by [18, Theorem 11.1.8], $\Phi$ and $\Phi_{\mid}$induce isomorphisms of Lie groups between $G L\left(d_{1}\right) \times \cdots \times G L\left(d_{l}\right) / N$ and $\otimes\left(G L\left(d_{1}\right), \ldots, G L\left(d_{l}\right)\right)$, and $O\left(d_{1}\right) \times \cdots \times O\left(d_{l}\right) / N$ and $\otimes\left(O\left(d_{1}\right), \ldots, O\left(d_{l}\right)\right)$, respectively.

Below, we describe the structure of the groups $G L_{\otimes}\left(\otimes_{i=1}^{l} \mathbb{R}^{d_{i}}\right)$ and $O_{\otimes}\left(\otimes_{i=1}^{l} \mathbb{R}^{d_{i}}\right)$. The case of tensor products of two spaces, i.e. $l=2$, was already established in [26, Proposition A.1].

Proposition A.4. $G L_{\otimes}\left(\otimes_{i=1}^{l} \mathbb{R}^{d_{i}}\right)$ and $O_{\otimes}\left(\otimes_{i=1}^{l} \mathbb{R}^{d_{i}}\right)$ are isomorphic (as Lie groups) to the semidirect product $\otimes\left(G L\left(d_{1}\right), \ldots, G L\left(d_{l}\right)\right) \rtimes \mathcal{P}$ and $\otimes\left(O\left(d_{1}\right), \ldots, O\left(d_{l}\right)\right) \rtimes \mathcal{P}$, respectively.

Proof. First observe that when only different integers are considered, i.e. $d_{i} \neq d_{j}$, for $i \neq j$, $i, j=1, \ldots, l$, then, by [25. Corollary 2.1.4], $G L_{\otimes}\left(\otimes_{i=1}^{l} \mathbb{R}^{d_{i}}\right)=\otimes\left(G L\left(d_{1}\right), \ldots, G L\left(d_{l}\right)\right)$, $O_{\otimes}\left(\otimes_{i=1}^{l} \mathbb{R}^{d_{i}}\right)=\otimes\left(O\left(d_{1}\right), \ldots, O\left(d_{l}\right)\right)$ and $\mathcal{P}=\left\{I_{\otimes_{i=1}^{l}} \mathbb{R}^{d_{i}}\right\}$. So in this case, the result is straightforward. 
In order to prove the general case, notice that from (2) in Lemma A.1, $G L_{\otimes}=$ $\otimes\left(G L\left(d_{1}\right), \ldots, G L\left(d_{l}\right)\right) \mathcal{P}$ and $\otimes\left(G L\left(d_{1}\right), \ldots, G L\left(d_{l}\right)\right) \cap \mathcal{P}$ is trivial. Therefore, we only need to check that $\otimes\left(G L\left(d_{1}\right), \ldots, G L\left(d_{l}\right)\right)$ is a closed normal subgroup of $G L_{\otimes}$, and that $G L_{\otimes}$ has a finite number of connected components. The first part of the result then follows from [18, Proposition 11.1.18].

By Lemma A.3, $\otimes\left(G L\left(d_{1}\right), \ldots, G L\left(d_{l}\right)\right)$ is a closed subgroup. To prove that it is a normal subgroup, it is enough to show that $U_{\sigma}\left(T_{1} \otimes \cdots \otimes T_{l}\right) U_{\sigma}^{-1} \in \otimes\left(G L\left(d_{1}\right), \ldots, G L\left(d_{l}\right)\right)$ for any $T_{i} \in G L\left(d_{i}\right)$ and $U_{\sigma} \in \mathcal{P}$. Let $x^{i} \in \mathbb{R}^{d_{i}}, i=1, \ldots, l$, then

$$
\begin{aligned}
U_{\sigma}\left(T_{1} \otimes \cdots \otimes T_{l}\right) U_{\sigma}^{-1}\left(x^{1} \otimes \cdots \otimes x^{l}\right) & =U_{\sigma} T_{1}\left(x^{\sigma^{-1}(1)}\right) \otimes \cdots \otimes T_{l}\left(x^{\sigma^{-1}(l)}\right) \\
& =T_{\sigma(1)}\left(x^{1}\right) \otimes \cdots \otimes T_{\sigma(l)}\left(x^{l}\right)=T_{\sigma(1)} \otimes \cdots \otimes T_{\sigma(l)}\left(x^{1} \otimes \cdots \otimes x^{l}\right) .
\end{aligned}
$$

Thus, $U_{\sigma}\left(T_{1} \otimes \cdots \otimes T_{l}\right) U_{\sigma}^{-1}=T_{\sigma(1)} \otimes \cdots \otimes T_{\sigma(l)}$ is a linear map on $\otimes\left(G L\left(d_{1}\right), \ldots, G L\left(d_{l}\right)\right)$, as required.

To prove that $G L_{\otimes}$ has a finite number of connected components, note that by Lemma A.1, the composition induces a continuous surjective map from $\otimes\left(G L\left(d_{1}\right), \ldots, G L\left(d_{l}\right)\right) \times$ $\mathcal{P}$ onto $G L_{\otimes}$. Hence, since both $\otimes\left(G L\left(d_{1}\right), \ldots, G L\left(d_{l}\right)\right)$ and $\mathcal{P}$ have a finite number of connected components, the same holds for $G L_{\otimes}$.

It remains to show the assertion for $O_{\otimes}$. By Lemma A.3. $\otimes\left(O\left(d_{1}\right), \ldots, O\left(d_{l}\right)\right)$ is closed. Also, since (A.2) is valid for $U_{i} \in O\left(d_{i}\right), i=1, \ldots, l$, then it is a closed normal subgroup of $O_{\otimes}$. In addition, by Lemma A.1 the composition map also gives us a surjective continuous map from $\otimes\left(O\left(d_{1}\right), \ldots, O\left(d_{l}\right)\right) \times \mathcal{P}$ onto $O_{\otimes}$. From this $O_{\otimes}$ has a finite number of connected components, and the result follows from [18, Proposition 11.1.18].

Corollary A.5. $G L_{\otimes}\left(\otimes_{i=1}^{l} \mathbb{R}^{d_{i}}\right) / O_{\otimes}\left(\otimes_{i=1}^{l} \mathbb{R}^{d_{i}}\right)$ is homeomorphic to $\mathbb{R}^{p}$ with $p=\frac{d_{1}\left(d_{1}+1\right)}{2}+$ $\cdots+\frac{d_{l}\left(d_{l}+1\right)}{2}$.

Proof. By Proposition A.4 $G L_{\otimes}$ has a finite number of connected components. Consequently, its quotient by $O_{\otimes}$, which is a maximal compact subgroup (Proposition A.2), must be homeomorphic to $\mathbb{R}^{p}$, [33, Theorem 32.5]. Therefore, from Lemma A.3 and Proposition A.4, we have $p=\frac{d_{1}\left(d_{1}+1\right)}{2}+\cdots+\frac{d_{l}\left(d_{l}+1\right)}{2}$.

The next lemma exhibits the polar decomposition of any linear isomorphism in $G L_{\otimes}$. It shows that every $T \in G L_{\otimes}$ can be written uniquely as $T=S U$ where $U$ is an orthogonal map in $O_{\otimes}$ and $S$ is the tensor product of strictly positive linear maps. Recall that a linear map $S: \mathbb{E} \rightarrow \mathbb{E}$, on a Euclidean space $\mathbb{E}$, is strictly positive if it is self-adjoint and $\langle x, T x\rangle_{\mathbb{E}}>0$ for every non-zero $x \in \mathbb{E}$.

Lemma A.6. Let $\mathcal{A}$ consists of tensor products $S_{1} \otimes \cdots \otimes S_{l}$ of strictly positive linear maps $S_{i} \in G L\left(d_{i}\right), i=1, \ldots, l$. Then:

1. $\mathcal{A} \subset G L_{\otimes}\left(\otimes_{i=1}^{l} \mathbb{R}^{d_{i}}\right)$ is closed.

2. The map $\Psi: \mathcal{A} \times O_{\otimes}\left(\otimes_{i=1}^{l} \mathbb{R}^{d_{i}}\right) \rightarrow G L_{\otimes}\left(\otimes_{i=1}^{l} \mathbb{R}^{d_{i}}\right)$ sending the pair $(S, U)$ to $S U$ is a homeomorphism.

Proof. (1). To show that $\mathcal{A}$ is closed, let $S_{1, n} \otimes \cdots \otimes S_{l, n}$ be a sequence in $\mathcal{A}$ converging to $S \in G L_{\otimes}$. Since $\otimes\left(G L\left(d_{1}\right), \ldots, G L\left(d_{l}\right)\right)$ is closed (Proposition A.3), and positive selfadjoint linear maps are stable under taking limits, then $S$ is a positive linear isomorphism (i.e. strictly positive). Moreover, it is of the form $S=T_{1} \otimes \cdots \otimes T_{l}$, for some $T_{i} \in G L\left(d_{i}\right)$, 
$i=1, \ldots, l$. Now, if $T_{i}=S_{i} U_{i}$ is the polar decomposition of $T_{i}$ (i.e. $S_{i}$ is strictly positive and $U_{i}$ is orthogonal), then $S=\left(S_{1} \otimes \cdots \otimes S_{l}\right)\left(U_{1} \otimes \cdots \otimes U_{l}\right)$. So, by the uniqueness of the polar decomposition $([21$, Theorem 60$]), U_{1} \otimes \cdots \otimes U_{l}$ must be the identity on $\otimes_{i=1}^{l} \mathbb{R}^{d_{i}}$. This shows that $S \in \mathcal{A}$ as desired.

(2). To show that $\Psi$ is bijective, let $T \in G L_{\otimes}$ then, by Lemma A.1 $T=T_{1} \otimes \cdots \otimes T_{l} U_{\sigma}$ for some $T_{i} \in G L\left(d_{i}\right)$ and $U_{\sigma} \in \mathcal{P}$. Also, as a consequence of the above argument, $T$ can be written as $T=\left(S_{1} \otimes \cdots \otimes S_{l}\right)\left(U_{1} \otimes \cdots \otimes U_{l}\right) U_{\sigma}$ and so $T=S U$, for $S=S_{1} \otimes \cdots \otimes S_{l} \in \mathcal{A}$ and $U=\left(U_{1} \otimes \cdots \otimes U_{l}\right) U_{\sigma} \in O_{\otimes}$. Indeed, since the polar decomposition of linear isomorphims is unique ([21, Theorem 60]) then so are $S, U$. Hence $\Psi$ is bijective. Its continiuty follows by definition. It reminds to show that $\Psi^{-1}$ is continuous. Let $T_{n}=\Psi\left(S_{n}, U_{n}\right)$ be a sequence converging to $T=\Psi(S, U)$. From the compactness of $O_{\otimes}$ and the fact that $\mathcal{A}$ is closed, it follows that $U_{n}$ converges to $U$ and $S_{n}$ to $S$. This shows that $\Psi^{-1}$ is continuous as required.

\section{References}

[1] H. Abels. Parallelizability of proper actions, global $K$-slices and maximal compact subgroups. Math. Ann., 212:1-19, 1974.

[2] S. Ageev and D. Repovš. On Banach-Mazur compacta. J. Austral. Math. Soc. Ser. A, 69(3):316-335, 2000.

[3] S. Antonyan. The topology of the Banach-Mazur compactum. Fundamenta Mathematicae, 166(3):209-232, 2000.

[4] S. Antonyan. West's problem on equivariant hyperspaces and Banach-Mazur compacta. Trans. Amer. Math. Soc., 355(8):3379-3404, 2003.

[5] S. Antonyan and N. Jonard-Pérez. Affine group acting on hyperspaces of compact convex subsets of $\mathbb{R}^{n}$. Fund. Math, 223:99-136, 2013.

[6] S. Antonyan, N. Jonard-Pérez, and S. Juárez-Ordóñez. Hyperspaces of convex bodies of constant width. Topology Appl., 196(part B):347-361, 2015.

[7] G. Aubrun and S. Szarek. Tensor products of convex sets and the volume of separable states on n qudits. Physical Review A, 73(2):022109, 2006.

[8] G. Aubrun and S. Szarek. Alice and Bob Meet Banach: The Interface of Asymptotic Geometric Analysis and Quantum Information Theory, volume 223. American Mathematical Soc., 2017.

[9] G. Bredon. Introduction to compact transformation groups. Academic Press, New York-London, 1972.

[10] V. De Silva and L-H. Lim. Tensor rank and the ill-posedness of the best low-rank approximation problem. SIAM J. Matrix Anal. and Appl., 30(3):1084-1127, 2008.

[11] A. Defant and K. Floret. Tensor Norms and Operator Ideals, volume 176. North Holland Mathematics Studies, 1st Edition edition, 1992.

[12] J. Diestel, J. Fourie, and J. Swart. The metric theory of tensor products. American Mathematical Society, Providence, RI, 2008. Grothendieck's résumé revisited. 
[13] M. Fernández-Unzueta. The Segre cone of Banach spaces and multilinear mappings. Linear Multilinear Algebra, 2018. DOI: 10.1080/03081087.2018.1509938.

[14] M. Fernández-Unzueta and L. Higueras-Montaño. A General Theory of Tensor Products of Convex Sets in Euclidean Spaces. arXiv:1906.11377. Submitted.

[15] M. Fernández-Unzueta and L. Higueras-Montaño. Convex Bodies Associated to Tensor Norms. J. Convex Anal., 26(4). Elect. publ. http://www.heldermann.de/JCA/JCA26/JCA264/jca26068.htm (to appear in print).

[16] E. Gluskin. The diameter of the Minkowski compactum is roughly equal to n. Funktsional. Anal. i Prilozhen., 15(1):72-73, 1981.

[17] A. Grothendieck. Résumé de la théorie métrique des produits tensoriels topologiques. Bol. Soc. Mat. São Paulo, 8:1-79, 1953.

[18] J. Hilgert and K. Neeb. Structure and geometry of Lie groups. Springer, New York, 2012 .

[19] F. John. Extremum problems with inequalities as subsidiary conditions. In Studies and Essays Presented to R. Courant on his 60th Birthday, January 8, 1948, pages 187-204. Interscience Publishers, Inc., New York, N. Y., 1948.

[20] R. Kadison and J. Ringrose. Fundamentals of the theory of operator algebras. Academic Press, 1983.

[21] I. Kaplansky. Linear Algebra and Geometry: a second course. Dover Publ., New York, 1974.

[22] S. Khot and A. Naor. Grothendieck-type inequalities in combinatorial optimization. Comm. Pure Appl. Math., 65(7):992-1035, 2012.

[23] T. Kolda and B. Bader. Tensor decompositions and applications. SIAM Rev., 51(3):455-500, 2009.

[24] J. Landsberg. Tensors: asymptotic geometry and developments 2016-2018. American Mathematical Society, Providence, RI, 2019.

[25] M-H. Lim. Additive preservers of non-zero decomposable tensors. Linear Algebra Appl., 428:239-253, 2008.

[26] T. Mettler. Reduction of $\beta$-integrable 2-Segre structures. Comm. Anal. Geom., 21(2):331-353, 2013.

[27] H. Minkowski. Geometrie der Zahlen. 1910. Teubner, Leipzig, 1927.

[28] R. Palais. The classification of G-spaces. Mem. Amer. Math. Soc. No. 36, 1960.

[29] R. Palais. On the existence of slices for actions of non-compact Lie groups. Ann. of Math. (2), 73:295-323, 1961.

[30] G. Pisier. Grothendieck's theorem, past and present. Bull. Amer. Math. Soc. (N.S.), 49(2):237-323, 2012. 
[31] R. Ryan. Introduction to tensor products of Banach spaces. Springer monographs in mathematics, 2002.

[32] R. Schneider. Convex bodies: the Brunn-Minkowski theory. Cambridge University Press, Cambridge, 1993.

[33] M. Stroppel. Locally compact groups. European Mathematical Society (EMS), Zürich, 2006.

[34] S. J. Szarek. On the geometry of the Banach-Mazur compactum. In E.E. Odwell and H.P. Rosenthal, editors, Functional Analysis, volume 1470. Springer, Berlin, 1991.

[35] N. Tomczak-Jaegermann. Banach-Mazur distances and finite-dimensional operator ideals. Longman Sc \& Tech, 1989. 